\title{
Exact solution for the extensional flow of a viscoelastic filament
}

\author{
LINDA B. SMOLKA ${ }^{1,2} \uparrow$, ANDREW BELMONTE $^{1}$, \\ DIANE M. HENDERSON ${ }^{1}$ and THOMAS P. WITELSKI ${ }^{2}$ \\ ${ }^{1}$ The W. G. Pritchard Fluid Mechanics Laboratory, Department of Mathematics, \\ Pennsylvania State University, University Park, PA 16802, USA \\ ${ }^{2}$ Department of Mathematics, Duke University, Box 90320, Durham, NC 27708, USA \\ email: smolka@bucknell.edu
}

(Received 24 November 2003; revised 16 June 2004)

\begin{abstract}
We solve the free boundary problem for the dynamics of a cylindrical, axisymmetric viscoelastic filament stretching in a gravity-driven extensional flow for the Upper Convected Maxwell and Oldroyd-B constitutive models. Assuming the axial stress in the filament has a spatial dependence provides the simplest coupling of viscoelastic effects to the motion of the filament, and yields a closed system of ODEs with an exact solution for the stretch rate and filament thickness satisfied by both constitutive models. This viscoelastic solution, which is a generalization of the exact solution for Newtonian filaments, converges to the Newtonian power-law scaling as $t \rightarrow \infty$. Based on the exact solution, we identify two regimes of dynamical behavior called the weakly- and strongly-viscoelastic limits. We compare the viscoelastic solution to measurements of the thinning filament that forms behind a falling drop for several semi-dilute (strongly-viscoelastic) polymer solutions. We find the exact solution correctly predicts the time-dependence of the filament diameter in all of the experiments. As $t \rightarrow \infty$, observations of the filament thickness follow the Newtonian scaling $1 / \sqrt{t}$. The transition from viscoelastic to Newtonian scaling in the filament thickness is coupled to a stretch-to-coil transition of the polymer molecules.
\end{abstract}

\section{Introduction}

The fall of a fluid droplet from a faucet under the influence of gravity is an everyday occurrence in kitchen sinks, and has recently received attention due to a series of related experimental studies $[37,43,54,30,7,21,25]$ and mathematical analyses [43, 7, 12, 41, $14,42,36,4,13,35,51,53,22]$ (see the review by Eggers [15]). While many studies have focused on the singularity occurring when the drop pinches off $[37,30,7,21,41,14,36,53]$, less attention has been paid to the long filament that is drawn out by the falling drop $[22,15,27]$. Herein, we derive an exact solution for the time-dependent stretch rate and filament thickness for a cylindrical, axisymmetric viscoelastic filament stretching in a gravity-driven extensional flow, and compare our predictions to measurements of the thinning filament that forms behind a falling drop.

$\dagger$ Current address: Department of Mathematics, Bucknell University, Lewisburg, PA, 17837, USA. 


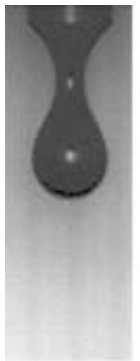

(a)

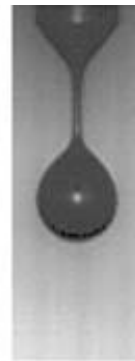

(b)

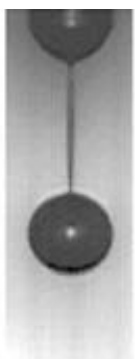

(c)

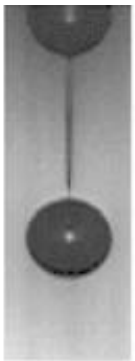

(d)

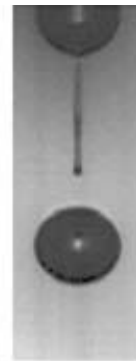

(e)

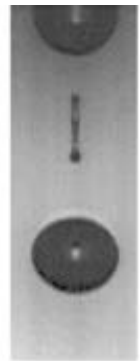

(f)

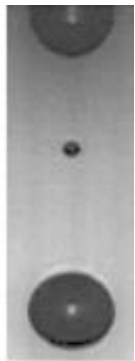

(g)

Figure 1. Evolution of a Newtonian drop of 80:20 glycerol/water falling in air from its formation to pinch-off and the formation of a satellite drop. The size of each image is $0.66 \times 1.94 \mathrm{~cm}^{2}$. The relative times of each image are (a) $0 \mathrm{~ms}$, (b) $14 \mathrm{~ms}$, (c) $20.8 \mathrm{~ms}$, (d) $22.2 \mathrm{~ms}$, (e) $23.5 \mathrm{~ms}$, (f) $25 \mathrm{~ms}$, (g) $33 \mathrm{~ms}$.

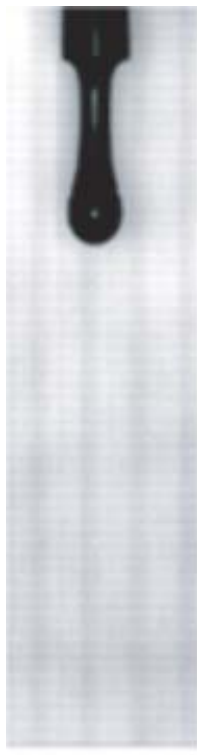

(a)

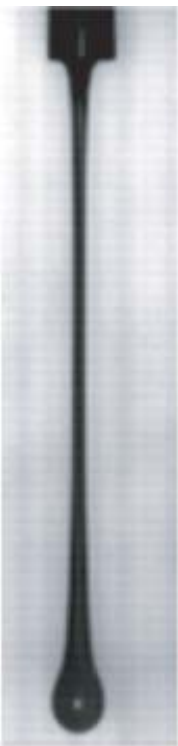

(b)

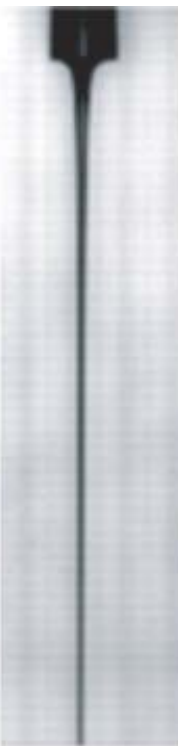

(c)

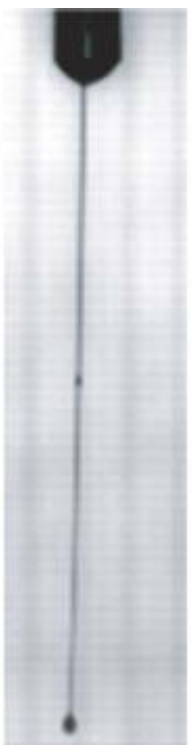

(d)

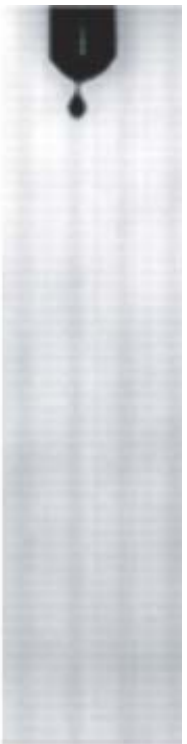

(e)

FIGURE 2. Evolution of a viscoelastic drop of $0.078 \%$ xanthan gum in $80: 20$ glycerol/water falling in air. The size of each image is $1.21 \times 4.82 \mathrm{~cm}^{2}$. The relative times of each image are (a) $0 \mathrm{~ms}$, (b) $815 \mathrm{~ms}$, (c) $988 \mathrm{~ms}$, (d) $1453 \mathrm{~ms}$, (e) $1492 \mathrm{~ms}$. The length of the drop (extending past the edge of the image) is approximately $1 \mathrm{~m}$ just before pinch-off at a time between frames (c) and (d).

Experiments illustrating the evolution of a droplet and the formation of this filament are shown in Figure 1 for a Newtonian fluid, and in Figure 2 for the same fluid with a small amount of the polymer xanthan gum added, causing it to have non-Newtonian behavior. Large differences in length and time scales are caused by this small change in the fluid composition. At the time of pinch-off, the filament length for the Newtonian solvent is $1.4 \mathrm{~cm}$ compared to approximately $1 \mathrm{~m}$ for the polymer solution, given the same experimental conditions [44]. In other experimental settings, as the fluid elasticity 
was increased by increasing the polymer concentration or molecular weight, the rate of filament thinning decreased [2], the time to filament breakup increased [3], and the breakup length of jets increased [33,9]. These observations each illustrate the ability of viscoelasticity to enhance the stability of filaments to capillary breakup. Understanding the motion and instabilities of liquid filaments and the consequences of non-Newtonian effects is important for the control of flow processes where applications include the manufacturing of fiber-optic cable (fiber drawing) and synthetic fibers, such as kevlar and lycra (fiber-spinning).

Modeling a liquid filament that is being stretched by a constant acceleration (or constant body force) is a challenging free boundary problem. Several studies have focused on the slender body limit, which reduces the dynamics to one dimension [43, 14, 42, 36, 4, 13, 35]. Similarity solutions describing the nonlinear motion of a Newtonian filament close to the pinch region were found by Papageorgiou [36] for Stokes flow; by Eggers [14], who included inertial effects; and by Zhang \& Lister [53], who considered pinch-off of a viscous thread surrounded by another viscous fluid. Recently, significant progress has been made in numerical simulations of the fully nonlinear, transient Navier-Stokes system solved in two dimensions, using a finite element method by Wilkes et al. [51]. Their numerical model predicts both the length of a drop, measured from the orifice to the end of the drop, at breakup and the volume of the primary drop (e.g. the larger drop in Figure 1(g)) for a range of parameters.

Frankel \& Weihs [17, 18] found an exact solution for the motion of a cylindrical, axisymmetric jet and considered its linear stability within the Navier-Stokes equations. Henderson et al. [22] applied this solution to the filament drawn out behind a falling drop and conducted a related stability analysis to obtain a prediction concerning the filament's breakup. The flow field for the exact solution is purely extensional with a stretch rate that is a decreasing function of time,

$$
\dot{\varepsilon}(t)=\frac{1}{t+t_{0}},
$$

and the filament radius follows

$$
h(t)=\frac{h_{0}}{\sqrt{1+t / t_{0}}},
$$

where $t_{0}$ is a positive constant and $h_{0}>0$ is the initial radius. This prediction for filament thickness was verified by the experiments of Henderson et al. [22] for Newtonian fluids with various values of viscosity and surface tension. In this paper, we will generalize this result for two types of linear viscoelastic models.

The dynamics of viscoelastic fluids are more complicated than that of Newtonian fluids since elastic (memory) and normal (forces perpendicular to the flow direction) effects can influence the flow, and the fluid properties themselves can vary with gradients in the flow. In extensional flows, the extensional viscosity is of importance [33,9]; for Newtonian fluids, it is three times the dynamic shear viscosity [49]. For viscoelastic fluids that strain-harden, the extensional viscosity can be up to 1000 times larger than the shear viscosity [47]. To account for these different "non-Newtonian" behaviors, numerous constitutive models have been developed for viscoelastic fluids, see Joseph [26] and Bird et al. [5]. Numerical 
studies have focused on the enhanced stability of viscoelastic filaments and jets. In their investigation of a model for a stretching viscoelastic filament, Markovich \& Renardy [32] showed that elasticity acts to stabilize the filament against surface tension effects, delaying its eventual breakup. And in numerical studies on the breakup of viscoelastic jets, Renardy showed an Oldroyd-B fluid, which develops infinite extensional stress at a finite stretch rate, never breaks, while a Giesekus fluid, whose extensional stress remains bounded for all stretch rates, breaks up in finite time [38].

Analytic studies of the flow in an Oldroyd-B liquid bridge predict that the radius decreases exponentially in time when capillary and elastic forces are balanced, and inertial and viscous effects are ignored; these assumptions are applied in the long time limit $[8,10]$. In this regime, the stretch rate of the flow is constant and the filament radius follows $h(t)=h_{0} \exp (-t /(3 \mathrm{De}))$, where the Deborah number is the ratio of the relaxation time and a characteristic flow time. Similar results were found by Entov \& Hinch [16], who considered the extensional flow of a FENE fluid with a spectrum of relaxation times. By balancing capillary and elastic forces at intermediate times, they predict the stretch rate approaches a constant and the filament radius decays quasi-exponentially in time. The exponential thinning of viscoelastic filaments has been observed in various experimental and numerical settings $[2,3,10]$, e.g. the thinning of a viscoelastic filament connected between large perturbations (known as 'beads-on-a-string') where the mass of the filament decreases as fluid drains into the beads [10].

To examine the effects of viscoelasticity on filament dynamics, we extend the results of Frankel \& Weihs [17, 18] and Henderson et al. [22] to a viscoelastic filament. In $\S 2$, we present an analytic formulation for the extensional flow of a cylindrical, axisymmetric filament falling under gravity through a quiescent fluid of negligible viscosity. Our approach provides a framework in which the known exact Newtonian solution can be generalized to various viscoelastic constitutive models using a condition for the existence of cylindrical solutions. We show that by assuming a power series expansion for the stress, the original free boundary problem, consisting of a system of partial differential equations (PDEs) and boundary conditions, is reduced to a system of coupled ordinary differential equations (ODEs) for the Upper Convected Maxwell and Oldroyd-B constitutive models. In this analysis, we allow inertial, viscous, elastic, gravitational and capillary forces to balance. The dependence of the unknowns on these forces is determined by the reduction of the free boundary problem to the system of ODEs under the constraints of an extensional flow in a cylindrical geometry.

An exact solution for any viscoelastic flow is unusual, yet for the Upper Convected Maxwell and Oldroyd-B constitutive models, this approach yields an analytic solution for the motion of the filament [45], as described in $\S 3$. This new solution allows us to analyze the effects of viscoelasticity on the filament motion; in particular, we distinguish between the weakly- and strongly-viscoelastic limits as we study the transient and long-time limits. In $\S 4$, we compare this analytic solution to experimental measurements of the thinning filament that forms behind a falling drop for several semi-dilute (strongly-viscoelastic) polymer solutions. Our solution predicts the time dependence of the filament radius which is in agreement with measurements. As $t \rightarrow \infty$ both the solution and the observations show the filament thickness scales as $1 / \sqrt{t}$, which is the scaling for a Newtonian fluid as in (1.2). This transition in the time dependence, from viscoelastic to Newtonian scaling, provides 
insight as to how the molecular dynamics couple to the filament's motion $[23,6,11,28]$ as explained in $\S 4$.

\section{Formulation of the free boundary problem}

Using the boundary value problem for the motion of an axisymmetric, cylindrical liquid filament stretching in a purely extensional flow, we derive a necessary condition for an exact solution to exist. This condition may be applied to either Newtonian or nonNewtonian fluids by introducing an appropriate constitutive relation, thereby closing the system of equations for the free boundary problem. The known exact Newtonian solution follows immediately from this condition [22]. Using the existence condition, we reduce the free boundary problem for the viscoelastic Upper Convected Maxwell and Oldroyd-B models to systems of coupled ODEs.

\subsection{Governing equations}

The governing equations for an incompressible fluid are the conservation of linear momentum and continuity equations

$$
\begin{aligned}
\rho\left[\frac{\partial \mathbf{u}}{\partial t}+(\mathbf{u} \cdot \nabla) \mathbf{u}\right] & =\nabla \cdot \mathbf{T}-\nabla p-\rho \mathbf{g}, \\
\nabla \cdot \mathbf{u} & =0,
\end{aligned}
$$

where $\rho$ is the density, $\mathbf{u}$ is the velocity, $\mathbf{T}$ is the symmetric extra stress tensor, $p$ is the pressure, and $\mathbf{g}=g \hat{\mathbf{e}}_{\mathbf{z}}$ is gravity with $\hat{\mathbf{e}}_{\mathbf{z}}$ directed upwards. For an axisymmetric flow $\left(u_{\theta} \equiv 0\right.$, $\left.\partial_{\theta}(\cdot) \equiv 0\right)$ the momentum equations in the $\hat{r}, \hat{\theta}$ and $\hat{z}$ directions are:

$$
\begin{aligned}
\frac{\partial u_{r}}{\partial t}+u_{r} \frac{\partial u_{r}}{\partial r}+u_{z} \frac{\partial u_{r}}{\partial z}+\frac{1}{\rho} \frac{\partial p}{\partial r} & =\frac{1}{\rho}\left(\frac{\partial T_{r r}}{\partial r}+\frac{T_{r r}}{r}+\frac{\partial T_{r z}}{\partial z}-\frac{T_{\theta \theta}}{r}\right), \\
0 & =\frac{\partial T_{r \theta}}{\partial r}+\frac{2 T_{r \theta}}{r}+\frac{\partial T_{\theta z}}{\partial z}, \\
\frac{\partial u_{z}}{\partial t}+u_{r} \frac{\partial u_{z}}{\partial r}+u_{z} \frac{\partial u_{z}}{\partial z}+\frac{1}{\rho} \frac{\partial p}{\partial z} & =\frac{1}{\rho}\left(\frac{\partial T_{r z}}{\partial r}+\frac{T_{r z}}{r}+\frac{\partial T_{z z}}{\partial z}\right)-g,
\end{aligned}
$$

in which the unknown functions depend on $r, z$, and $t$. We consider axisymmetric motions of a filament possessing an extensional velocity field

$$
\mathbf{u} \equiv\left(u_{r}, u_{\theta}, u_{z}\right)=\left(-\frac{1}{2} \dot{\varepsilon}(t) r, 0, \dot{\varepsilon}(t) z+v(t)\right),
$$

where $\dot{\varepsilon}(t)$ is the stretch rate and $v(t)$ is an additional uniform velocity necessitated by the presence of gravity [22]. We note that (2.4) satisfies the continuity equation (2.2). The symmetric part of the velocity gradient tensor, $\mathbf{D}=\left(\nabla \mathbf{u}+\nabla \mathbf{u}^{T}\right) / 2$ for this extensional flow is diagonal with

$$
D_{r r}=-\frac{1}{2} \dot{\varepsilon}=D_{\theta \theta}, \quad D_{z z}=\dot{\varepsilon} .
$$

The relationship between the stress field $\mathbf{T}$ and the flow field is prescribed by a constitutive model. Equations (2.3) can be simplified further, if the constitutive model 
under consideration is materially frame indifferent [39], that is, the stress is invariant under a change of coordinate system and under translations and rotations of fluid elements [5]. Following Renardy [39], the stress field associated with an extensional velocity field in rectangular coordinates ${ }^{1}$ is diagonal, which means the stress is shear free [39]. Under the Jacobian transformation from rectangular to cylindrical coordinates, we can conclude that $T_{r z}=0$ and $T_{\theta z}=0$ provided the stress is materially frame indifferent; this will be true of the constitutive models considered here. Given the second result, it follows from the azimuthal momentum equation $(2.3 \mathrm{~b})$ that $T_{r \theta}=0$, otherwise $T_{r \theta}$ diverges along the filament axis $(r=0)$, which is unphysical. Therefore, the stress field is diagonal in cylindrical coordinates, so that hereafter

$$
\mathbf{T}=\left(\begin{array}{ccc}
T_{r r} & 0 & 0 \\
0 & T_{\theta \theta} & 0 \\
0 & 0 & T_{z z}
\end{array}\right)
$$

The generalized form of the constitutive relations we consider is given by the materially frame indifferent Oldroyd-B model. The Oldroyd-B model is one of the simplest nonNewtonian models derivable from a simplified molecular theory [31]. The model, which includes stress contributions from the Newtonian solvent (viscous stress) and the polymer molecules (elastic relaxation), is given by

$$
\mathbf{T}+\lambda \frac{\hat{d} \mathbf{T}}{\hat{d t}}=2 \eta\left(\mathbf{D}+\lambda_{r} \frac{\hat{d} \mathbf{D}}{\hat{d t}}\right),
$$

where $\eta>0$ is the constant dynamic shear viscosity, $\lambda>0$ is the elastic relaxation time, $\lambda_{r}>0$ is the retardation time and the tensor derivative $\hat{d} / \hat{d} t$ is the upper convected derivative $[31,5]$

$$
\frac{\hat{d} \mathbf{X}}{\hat{d} t}=\left(\frac{\partial}{\partial t}+\mathbf{u} \cdot \nabla\right) \mathbf{X}-\nabla \mathbf{u}^{T} \cdot \mathbf{X}-\mathbf{X} \cdot \nabla \mathbf{u} .
$$

This model does not have shear-thinning or thickening effects. However, it does develop infinite extensional stresses, and thus infinite extensional viscosity, in steady extensional flow as the stretch rate approaches $\dot{\varepsilon}=1 / 2 \lambda[5,39]$. We will also consider two models that are simplifications of (2.7): (i) a Newtonian fluid ( $\lambda=0$ and $\lambda_{r}=0$ ), and (ii) an Upper Convected Maxwell fluid $\left(\lambda_{r}=0\right)$.

The boundary of the liquid filament is a free surface, which we denote by $r=h(z, t)$, as shown in Figure 3. The fluid surrounding the filament is assumed to be at rest and have negligible viscosity. At the interface, $r=h$, there are three boundary conditions (simplified for an axisymmetric flow):

(i) the kinematic condition,

$$
\partial_{t} h+u_{z} \partial_{z} h=u_{r}
$$

${ }^{1} \mathbf{u} \equiv\left(u_{x}, u_{y}, u_{z}\right)=\left(-\frac{1}{2} \dot{\varepsilon} x,-\frac{1}{2} \dot{\varepsilon} y, \dot{\varepsilon} z+v\right)$. 


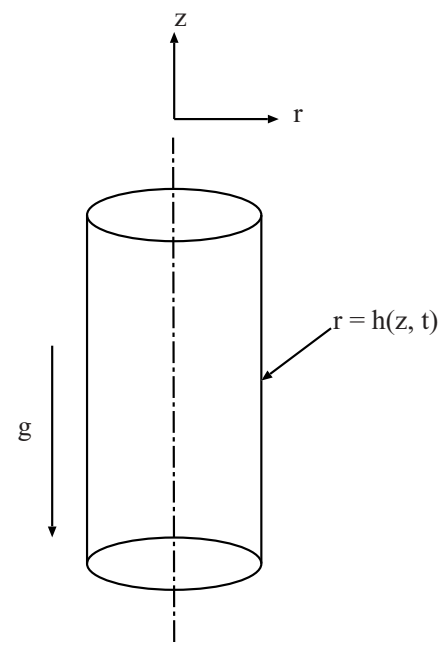

FigURE 3. Schematic of an axisymmetric cylindrical liquid filament.

(ii) the normal stress condition,

$$
p-\frac{T_{r r}-2 T_{r z} \partial_{z} h+T_{z z}\left(\partial_{z} h\right)^{2}}{1+\left(\partial_{z} h\right)^{2}}=\gamma\left(\frac{1}{h \sqrt{1+\left(\partial_{z} h\right)^{2}}}-\frac{\partial_{z z} h}{\left(1+\left(\partial_{z} h\right)^{2}\right)^{3 / 2}}\right)+p_{\mathrm{amb}},
$$

where $\gamma$ is the surface tension and $p_{\mathrm{amb}}$ is the constant pressure of the ambient fluid, and (iii) the tangential stress condition,

$$
\left(T_{r r}-T_{z z}\right) \partial_{z} h+T_{r z}\left(1-\left(\partial_{z} h\right)^{2}\right)=0 .
$$

We assume that the filament is cylindrical,

$$
\partial_{z} h=0 \quad \rightarrow \quad h=h(t)
$$

In Figure 2 for times between frames a-d, this assumption applies to large portions of the filament behind the falling drop. Under this assumption and using the extensional flow (2.4), the kinematic condition reduces to

$$
\frac{d h}{d t}=-\frac{\dot{\varepsilon} h}{2},
$$

the normal stress condition reduces to

$$
p(h, z, t)-T_{r r}(h, z, t)=\frac{\gamma}{h}+p_{\mathrm{amb}},
$$

and the tangential stress condition yields

$$
T_{r z}(h, z, t)=0 .
$$

Note that (2.13) is consistent with the earlier result on the form of the stress tensor (2.6). 
The governing equations (2.2)-(2.3) along with the boundary conditions (2.11)-(2.13) define the free boundary problem for a cylindrical filament (2.10) of a general fluid in an axisymmetric extensional flow (2.4). The constitutive model (2.7) we consider restricts the problem to a Newtonian, Upper Convected Maxwell, or Oldroyd-B fluid, depending on the choices of $\lambda$ and $\lambda_{r}$. A solution to this free boundary problem consists of the velocity field $\mathbf{u}$ (given in terms of $\dot{\varepsilon}$ and $v$ ), pressure field $p$, extra stress field $\mathbf{T}$, and filament radius $h$; the solution includes inertial, viscous, gravitational, capillary and elastic (if $\lambda \neq 0$ ) effects.

\subsection{Simplification of the free boundary problem}

Before incorporating a particular constitutive relation, we first develop a general condition, using (2.4), (2.3c), (2.10) and (2.12), that must be satisfied for a solution to exist to this free boundary problem. This condition is independent of the material properties of the fluid, therefore, it applies to any fluid model.

We begin by considering the normal stress condition (2.12) at the interface. Since $h$ is independent of $z$, this condition can be written as

$$
\frac{\partial}{\partial z}\left[p(h, z, t)-T_{r r}(h, z, t)\right]=0
$$

which does not depend on the surface tension $\gamma$. Substituting the velocity field (2.4) into the axial momentum equation $(2.3 c)$ we obtain

$$
z\left(\frac{d \dot{\varepsilon}}{d t}+\dot{\varepsilon}^{2}\right)+\frac{d v}{d t}+v \dot{\varepsilon}+\frac{1}{\rho} \frac{\partial p}{\partial z}=\frac{1}{\rho} \frac{\partial T_{z z}}{\partial z}-g
$$

which is valid for $0 \leqslant r \leqslant h$. Substituting $\partial p / \partial z$ from (2.15) into (2.14) leads to the condition

$$
-\rho z\left(\frac{d \dot{\varepsilon}}{d t}+\dot{\varepsilon}^{2}\right)-\rho\left(\frac{d v}{d t}+v \dot{\varepsilon}+g\right)+\frac{\partial T_{z z}}{\partial z}-\frac{\partial T_{r r}}{\partial z}=0,
$$

at the filament interface. This equation, when coupled with the appropriate constitutive model, provides a general condition for the existence of solutions.

Before proceeding, we note that the pressure can also be determined from the free boundary problem. We begin by substituting the velocity field (2.4) into the radial momentum equation $(2.3 a)$ to obtain an expression for the pressure gradient

$$
\frac{\partial p}{\partial r}=\frac{\rho r}{2}\left(\frac{d \dot{\varepsilon}}{d t}-\frac{\dot{\varepsilon}^{2}}{2}\right)+\left(\frac{\partial T_{r r}}{\partial r}+\frac{T_{r r}}{r}-\frac{T_{\theta \theta}}{r}\right) .
$$

Integrating with respect to $r$ leads to an equation for the pressure

$$
p(r, z, t)=\frac{\rho r^{2}}{4}\left(\frac{d \dot{\varepsilon}}{d t}-\frac{\dot{\varepsilon}^{2}}{2}\right)+T_{r r}(r, z, t)-T_{r r}(0, z, t)+\int_{0}^{r}\left(\frac{T_{r r}}{\tilde{r}}-\frac{T_{\theta \theta}}{\tilde{r}}\right) d \tilde{r}+p(0, z, t) .
$$

To find the pressure along the filament axis, $p(0, z, t)$, we substitute the pressure from (2.17) into $\partial p / \partial z$ in (2.15), and integrate with respect to $z$. Substituting the expression for 
$p(0, z, t)$ back into (2.17) leads to

$$
\begin{aligned}
p(r, z, t)=\frac{\rho r^{2}}{4}\left(\frac{d \dot{\varepsilon}}{d t}-\frac{\dot{\varepsilon}^{2}}{2}\right) & -\frac{\rho z^{2}}{2}\left(\frac{d \dot{\varepsilon}}{d t}+\dot{\varepsilon}^{2}\right)-\rho z\left(\frac{d v}{d t}+v \dot{\varepsilon}+g\right) \\
& +\int_{0}^{z} \frac{\partial T_{z z}}{\partial \tilde{z}} d \tilde{z}+G(r, t)+p(0,0, t)
\end{aligned}
$$

where several of the $z$-independent stress terms are combined into the function

$$
G(r, t)=\int_{0}^{r}\left(\frac{1}{\tilde{r}} \frac{\partial}{\partial \tilde{r}}\left(\tilde{r} T_{r r}(\tilde{r}, 0, t)\right)-\frac{T_{\theta \theta}}{\tilde{r}}(\tilde{r}, 0, t)\right) d \tilde{r} .
$$

We use this expression to find the pressure associated with the exact solution for various constitutive models.

To summarize, (2.16) represents a reduced form of the free boundary problem that is restricted to the filament interface and is the existence condition that needs to be satisfied by any fluid model. Next, we make simplifying assumptions on the stress field to differentiate between Newtonian and viscoelastic filament dynamics. These assumptions set the stage to reduce the free boundary problem for a viscoelastic fluid to a coupled system of ODEs.

\subsection{Assumptions on the stress field}

Evidence from experiments suggests that the motion of Newtonian and viscoelastic filaments are vastly different $[33,9,20,44,2,3]-$ see Figures 1 and 2. To reflect the change in the filament behavior for a viscoelastic fluid, one would expect the time dependence of the flow field, given by $\dot{\varepsilon}(t)$, to be different than in the Newtonian case. The dependence of the interfacial motion $h(t)$ on $\dot{\varepsilon}(t)$ in the kinematic condition (2.11) motivates this observation and leads us to the following conclusion: To obtain solutions for more general classes of constitutive models that are distinct from the Newtonian solution requires introducing $z$-dependence in at least one of the stress components $T_{r r}$ or $T_{z z}$. This follows immediately from the existence condition (2.16), since the stress of a Newtonian fluid is spatially homogeneous. We expect the dominant stress in an extensional flow of a cylindrical filament to be in the axial direction. Thus we look for solutions of $T_{z z}$ with a power series expansion of the form

$$
T_{z z}(h, z, t)=\sum_{n=0}^{\infty} c_{n}(t) z^{n}
$$

and we impose

$$
\frac{\partial T_{r r}}{\partial z}(h, z, t)=0
$$

In the Newtonian case, $c_{n}=0$ for $n \geqslant 1$ and (2.21) is true. Substituting (2.20) and (2.21) into the existence condition (2.16) yields

$$
-\rho z\left(\frac{d \dot{\varepsilon}}{d t}+\dot{\varepsilon}^{2}\right)-\rho\left(\frac{d v}{d t}+v \dot{\varepsilon}+g\right)+\sum_{n=1}^{\infty} n c_{n} z^{n-1}=0 .
$$


Collecting terms in powers of $z$ results in the following set of ordinary differential equations:

$$
\begin{gathered}
z^{0}: \quad-\rho\left(\frac{d v}{d t}+v \dot{\varepsilon}+g\right)+c_{1}=0, \\
z^{1}: \quad-\rho\left(\frac{d \dot{\varepsilon}}{d t}+\dot{\varepsilon}^{2}\right)+2 c_{2}=0, \\
z^{n-1}: \quad n c_{n}=0, \quad \text { for } \quad n \geqslant 3 .
\end{gathered}
$$

Since $c_{n}=0$ for $n \geqslant 3$, only a quadratic expansion for $T_{z z}$ is needed to modify the time dependence of the flow via $\dot{\varepsilon}(t)$ and $v(t)$. For convenience we re-write the expansion of $T_{z z}$ as

$$
T_{z z}(h, z, t)=\frac{1}{2} a(t) z^{2}+b(t) z+c(t) .
$$

This expression provides the simplest coupling of a viscoelastic model to the dynamics of an axisymmetric, cylindrical filament through (2.16).

The complete solution of the free boundary problem includes the stress and pressure fields. Solving for the stress field explicitly inside the filament $(0 \leqslant r<h)$ with either the Upper Convected Maxwell or Oldroyd-B models is non-trivial since these models (2.7) are linear partial differential equations that require boundary and initial conditions. The situation is simpler for a Newtonian fluid, where the stress depends only on time. Since it is not clear what the appropriate boundary conditions are in the viscoelastic case, we make the following assumptions to provide the simplest connection between the Newtonian and viscoelastic cases, namely:

- The axial stress $T_{z z}$ does not vary radially, thus by continuity (2.24) is valid for $0 \leqslant r \leqslant h$.

- The radial and azimuthal stress, $T_{r r}$ and $T_{\theta \theta}$, do not vary in $r$ or $z$.

Given that $T_{r r}$ and $T_{\theta \theta}$ are independent of $r$ implies that $T_{r r}=T_{\theta \theta}$, otherwise the pressure is unbounded (see (2.18) and (2.19)). These simplifying assumptions are consistent with the above analysis and are needed only to estimate the stress and pressure fields.

\subsection{Newtonian fluid}

Here we review the known exact solution for a Newtonian filament [22] by using the existence condition (2.16). The constitutive law for a Newtonian fluid is the simplest symmetric relation between the stress field and gradients in the flow, namely

$$
\mathbf{T}=2 \eta \mathbf{D} \text {. }
$$

For the extensional flow (2.4), this model yields $\mathbf{T}$ diagonal and spatially uniform with $T_{r r}=-\eta \dot{\varepsilon}=T_{\theta \theta}$ and $T_{z z}=2 \eta \dot{\varepsilon}$. This is a trivial case of (2.24) with $a=b=0$ and $c(t)=2 \eta \dot{\varepsilon}(t)$. Substituting $T_{r r}$ and $T_{z z}$ into (2.16), then collecting terms in powers of $z$ results in two coupled ODEs:

$$
\begin{gathered}
\frac{d \dot{\varepsilon}}{d t}+\dot{\varepsilon}^{2}=0 \\
\frac{d v}{d t}+v \dot{\varepsilon}=-g .
\end{gathered}
$$


These can be solved exactly, with solutions

$$
\dot{\varepsilon}(t)=\frac{1}{t+t_{0}} \quad \text { and } \quad v(t)=-\frac{g}{2}\left(t+t_{0}\right)+\frac{z_{0}}{t+t_{0}},
$$

where $t_{0}=\dot{\varepsilon}(0)^{-1}$ and $z_{0}=v(0) t_{0}+\frac{1}{2} g t_{0}^{2}$, and thus the velocity components (2.4) are

$$
u_{r}=-\frac{r}{2\left(t+t_{0}\right)} \quad \text { and } \quad u_{z}=\frac{z+z_{0}}{t+t_{0}}-\frac{g}{2}\left(t+t_{0}\right)
$$

which satisfy the continuity equation, $\nabla \cdot \mathbf{u}=\partial_{r}\left(r u_{r}\right) / r+\partial_{z}\left(u_{z}\right)=0$. Using the kinematic condition (2.11), we find the filament interface is given by

$$
h(t)=\frac{h(0)}{\sqrt{1+t / t_{0}}} .
$$

The pressure is found using (2.18) and (2.19) along with the normal stress condition (2.12) resulting in

$$
p(r, t)=\frac{3}{8} \rho \frac{h^{2}-r^{2}}{\left(t+t_{0}\right)^{2}}-\frac{\eta}{t+t_{0}}+\frac{\gamma}{h}+p_{\mathrm{amb}},
$$

which is independent of $z$. This solution is valid for viscous $(\eta>0)$ or inviscid $(\eta=0)$ fluids, with or without surface tension $(\gamma)$, and with or without gravity $(g)$. The case with $g=0$ is the exact solution found by Frankel \& Weihs [17]. The case with $g>0$ is the exact solution found by Henderson et al. [22] in the present notation where, in particular, our $t_{0}$ is their $t^{*}$. The solution with $g>0$ has a simple interpretation when viewed in Lagrangian coordinates, that is, it describes the free fall of a liquid filament in a uniform gravitational field [22]. Further, Henderson et al. showed that: (i) (2.29) predicts well their observations of the thinning filament drawn out by drops of viscous, Newtonian fluids, and (ii) criterion developed from a linear stability analysis of this exact solution correctly predict the manner in which the filament breaks up in experiments [22].

It is worth noting that the functional forms of the stretch rate and filament radius depend only on the inertia of the flow (see (2.26a) and (2.11)), and are monotonically decreasing functions of time.

As an aside, although this exact solution was not derived in the slender body limit, it does satisfy the slender body equations for a Newtonian fluid jet $[12,15]$ :

$$
\begin{aligned}
\frac{\partial\left(h^{2}\right)}{\partial t}+\frac{\partial\left(h^{2} u_{z}\right)}{\partial z} & =0 \\
\frac{\partial u_{z}}{\partial t}+u_{z} \frac{\partial u_{z}}{\partial z} & =-\frac{\gamma}{\rho h^{2}} \frac{\partial h}{\partial z}+\frac{3 \eta}{\rho h^{2}} \frac{\partial}{\partial z}\left(h^{2} \frac{\partial u_{z}}{\partial z}\right)-g
\end{aligned}
$$

Next we specialize our analysis of the motion of a cylindrical filament in an axisymmetric extensional flow to the Upper Convected Maxwell and Oldroyd-B constitutive models, highlighting similarities in the solutions of these two viscoelastic models. 


\subsection{Upper convected Maxwell fluid}

Here we consider the consequences of the existence condition (2.16) when the axial stress component is quadratic in $z$ as discussed in $\S 2.2$ and $\S 2.3$, for an Upper Convected Maxwell fluid. The Upper Convected Maxwell (UCM) constitutive model is given by

$$
\mathbf{T}+\lambda \frac{\hat{d} \mathbf{T}}{\hat{d t}}=2 \eta \mathbf{D}
$$

where $\lambda$ is the relaxation time and the tensor derivative is described by (2.8).

Using (2.32) and (2.8) with the extensional field (2.4) results in an axial stress that obeys

$$
T_{z z}+\lambda\left(\frac{\partial T_{z z}}{\partial t}+(\dot{\varepsilon} z+v) \frac{\partial T_{z z}}{\partial z}-2 \dot{\varepsilon} T_{z z}\right)=2 \eta \dot{\varepsilon}
$$

Substituting the quadratic expansion for $T_{z z}$ (2.24) into the conditional statement (2.16) and the constitutive law (2.33) yields

$$
-\rho z\left(\frac{d \dot{\varepsilon}}{d t}+\dot{\varepsilon}^{2}\right)-\rho\left(\frac{d v}{d t}+v \dot{\varepsilon}+g\right)+a z+b=0,
$$

and

$$
\lambda\left[\frac{1}{2} \frac{d a}{d t} z^{2}+\frac{d b}{d t} z+\frac{d c}{d t}+(\dot{\varepsilon} z+v)(a z+b)-2 \dot{\varepsilon}\left(\frac{a}{2} z^{2}+b z+c\right)\right]+\frac{a}{2} z^{2}+b z+c=2 \eta \dot{\varepsilon} .
$$

Collecting terms in powers of $z$ results in a set of five coupled ODEs:

$$
\begin{aligned}
\frac{d \dot{\varepsilon}}{d t}+\dot{\varepsilon}^{2} & =\frac{1}{\rho} a, \\
\frac{d v}{d t}+v \dot{\varepsilon} & =\frac{1}{\rho} b-g, \\
\lambda \frac{d a}{d t}+a & =0 \\
\lambda \frac{d b}{d t}+(1-\lambda \dot{\varepsilon}) b & =-\lambda a v, \\
\lambda \frac{d c}{d t}+(1-2 \lambda \dot{\varepsilon}) c & =2 \eta \dot{\varepsilon}-\lambda b v .
\end{aligned}
$$

Solutions of this system of equations define the velocity field and axial stress. The pressure, which is computed using (2.12), (2.18) and (2.19), is given by

$$
p(r, t)=\frac{\rho}{4}\left(h^{2}-r^{2}\right)\left(\frac{3 \dot{\varepsilon}^{2}}{2}-\frac{a}{\rho}\right)+T_{r r}(t)+\frac{\gamma}{h}+p_{\mathrm{amb}},
$$

where the radial stress, defined by (2.32) and (2.8) with the extensional field (2.4), satisfies

$$
\lambda \frac{d T_{r r}}{d t}+(1+\lambda \dot{\varepsilon}) T_{r r}=-\eta \dot{\varepsilon}
$$


The solution for an axisymmetric Upper Convected Maxwell filament stretching in a gravitational field requires solving the coupled system of ODEs given by (2.36), (2.38) and (2.11).

\subsection{Oldroyd-B fluid}

Here we consider the consequences of the existence condition and the quadratic axial stress for an Oldroyd-B fluid, and highlight similarities with the UCM case. The OldroydB constitutive model, given by

$$
\mathbf{T}+\lambda \frac{\hat{d} \mathbf{T}}{\hat{d t}}=2 \eta\left(\mathbf{D}+\lambda_{r} \frac{\hat{d} \mathbf{D}}{\hat{d t}}\right)
$$

and the tensor derivative (2.8), is a generalization of the UCM model in which the additional term, $2 \eta \lambda_{r}(\hat{d} \mathbf{D} / \hat{d t})$, arises from stresses in the solvent [31].

Using (2.39) and (2.8) with the extensional field (2.4) results in an axial stress that obeys

$$
T_{z z}+\lambda\left(\frac{\partial T_{z z}}{\partial t}+(\dot{\varepsilon} z+v) \frac{\partial T_{z z}}{\partial z}-2 \dot{\varepsilon} T_{z z}\right)=2 \eta\left(\dot{\varepsilon}+\lambda_{r}\left(\frac{d \dot{\varepsilon}}{d t}-2 \dot{\varepsilon}^{2}\right)\right) .
$$

Substituting the quadratic expansion for $T_{z z}$ (2.24) into the existence condition (2.16) and the constitutive law (2.40) yields (2.34) and

$$
\begin{array}{r}
\lambda\left[\frac{1}{2} \frac{d a}{d t} z^{2}+\frac{d b}{d t} z+\frac{d c}{d t}+(\dot{\varepsilon} z+v)(a z+b)-2 \dot{\varepsilon}\left(\frac{a}{2} z^{2}+b z+c\right)\right] \\
+\frac{a}{2} z^{2}+b z+c=2 \eta\left[\dot{\varepsilon}+\lambda_{r}\left(\frac{d \dot{\varepsilon}}{d t}-2 \dot{\varepsilon}^{2}\right)\right] .
\end{array}
$$

Collecting terms in powers of $z$ results in five coupled ODEs:

$$
\begin{aligned}
\frac{d \dot{\varepsilon}}{d t}+\dot{\varepsilon}^{2} & =\frac{1}{\rho} a, \\
\frac{d v}{d t}+v \dot{\varepsilon} & =\frac{1}{\rho} b-g, \\
\lambda \frac{d a}{d t}+a & =0, \\
\lambda \frac{d b}{d t}+(1-\lambda \dot{\varepsilon}) b & =-\lambda a v, \\
\lambda \frac{d c}{d t}+(1-2 \lambda \dot{\varepsilon}) c & =2 \eta\left(\dot{\varepsilon}+\frac{\lambda_{r}}{\rho} a-3 \lambda_{r} \dot{\varepsilon}^{2}\right)-\lambda b v .
\end{aligned}
$$

The radial stress, defined by (2.39) and (2.8) with the extensional field (2.4), satisfies

$$
\lambda \frac{d T_{r r}}{d t}+(1+\lambda \dot{\varepsilon}) T_{r r}=-\eta\left(\dot{\varepsilon}+\frac{\lambda_{r}}{\rho} a\right)
$$

Note that if $\lambda_{r}=0$, then the Oldroyd-B model reduces to the UCM model equations (2.36) and (2.38). Further, if $\lambda=0$, then $a=b=0$ and $c=2 \eta \dot{\varepsilon}$, which is the Newtonian model for 
stress. The system of equations for the UCM and Oldroyd-B models are generalizations of the Newtonian model equations solved by Frankel \& Weihs with $g=0$ [17], and Henderson et al. with $g \neq 0$ [22].

This system of ODEs differs from (2.36) and (2.38) for the UCM model only in the equations for $c(2.42 e)$ and $T_{r r}(2.42 f)$. This difference arises from the additional solvent stress in (2.7), which contributes only time-dependent effects. As a consequence, the general solutions for the stretch rate $\dot{\varepsilon}$, the axial velocity component $v$ (and thus the velocity field $\mathbf{u}$ ), and the stress coefficients, $a$ and $b$, are identical for these two constitutive models. In addition, the solution of the filament thickness $h$, which depends on $\dot{\varepsilon}(2.11)$, is also shared by these two models. Differences arise in the stress component $T_{z z}$ via coefficient $c$, and in the pressure field by $T_{r r}$ (see (2.37)).

To summarize, we have reduced the free boundary problems for the Upper Convected Maxwell and Oldroyd-B fluids to two coupled systems of ODEs. In the next section, we nondimensionalize the more general Oldroyd-B system of equations, and determine exact solutions for the nondimensional forms of $a, \dot{\varepsilon}$ and $h$. We also discuss limiting forms of the viscoelastic solution and show that it is a generalization of the known Newtonian solution.

\section{Analysis of the model}

Since the governing equations for the Oldroyd-B filament (2.42) include the Newtonian and UCM models as special cases, we will focus our analysis on this system. In particular, questions of interest will center on describing the influence of elasticity.

Consider the limit of short elastic time scales, $\lambda \rightarrow 0$. For the limit $\lambda \rightarrow 0$ with time fixed, equations $(2.42 c, 2.42 d)$ trivially yield $a=b=0$. Consequently, for times long compared with $\lambda$, the stress becomes homogeneous in space and the filament stretch rate and velocity are given by the Newtonian model $(2.26 a, 2.26 b)$. From $(2.42 e)$, the stress is $c=2 \eta \dot{\varepsilon}\left(1-3 \lambda_{r} \dot{\varepsilon}\right)$ and converges to the Newtonian stress as $\dot{\varepsilon} \rightarrow 0$ for $t \rightarrow \infty$. This simple very-long time solution can be viewed as the outer solution of system $(2.42)$ as $\lambda \rightarrow 0$. To analyze the nature of the viscoelastic characteristics of the fluid filament, we will carefully examine the short-time behavior of the solutions, when time scales with $\lambda$; this scaling effectively defines an inner solution or initial layer.

\subsection{Non-dimensional equations}

We non-dimensionalize the Oldroyd-B filament equations using the scalings

$$
\begin{array}{clrl}
\tau=\frac{t}{\lambda}, & E=\lambda \dot{\varepsilon}, & V=\frac{v}{g \lambda}, & H=\frac{h}{h_{0}}, \\
A=\frac{a}{a_{0}}, & B=\frac{b}{\rho g}, & C=\frac{\lambda}{\eta} c, & \bar{T}_{r r}=\frac{\lambda}{\eta} T_{r r},
\end{array}
$$

where $a_{0}, h_{0}$ are the initial values of the quadratic axial stress coefficient and the filament radius, respectively. In particular, we use the elastic relaxation time $\lambda$ to set the time scale and gravitational acceleration to set the length $\left(L=g \lambda^{2}\right)$ and velocity $(U=g \lambda)$ scales. This 
choice results in the non-dimensional form of equations (2.42) and (2.11)

$$
\begin{gathered}
\frac{d E}{d \tau}+E^{2}=\frac{1}{4} \delta^{2} A, \\
\frac{d V}{d \tau}+V E=B-1, \\
\frac{d A}{d \tau}+A=0, \\
\frac{d B}{d \tau}+(1-E) B=-\frac{1}{4} \delta^{2} A V, \\
\frac{d C}{d \tau}+(1-2 E) C=2 E+\omega\left(\frac{1}{2} \delta^{2} A-6 E^{2}\right)-\psi B V, \\
\frac{d \bar{T}_{r r}+(1+E) \bar{T}_{r r}=-\left(E+\frac{1}{4} \delta^{2} \omega A\right),}{d \tau}=-\frac{1}{2} E H .
\end{gathered}
$$

The nondimensional system depends on three dimensionless parameters: (i) the Deborah number,

$$
\delta=2 \lambda \sqrt{a_{0} / \rho}=\mathrm{De},
$$

which compares the relaxation time of the fluid to a characteristic stretch rate $\dot{\varepsilon}_{\mathrm{c}}=\sqrt{a_{0} / \rho}$ that depends on the initial axial stress; (ii) the Bond number,

$$
\psi=\frac{\rho g^{2} \lambda^{3}}{\eta}=\frac{\mathrm{Re}}{\mathrm{Fr}^{2}}=\text { Bo, }
$$

where the Reynolds number $\operatorname{Re}=\rho U L / \eta$, compares inertial to viscous effects, and the Froude number $\operatorname{Fr}=U / \sqrt{g L}$, compares the velocity scale with the speed of shallow-water gravity waves [12]; and (iii) the ratio of retardation to relaxation times

$$
\omega=\frac{\lambda_{r}}{\lambda}
$$

Given our choice of scalings $\mathrm{Fr}=1$, and $\psi$ is simply the Reynolds number. Under these scalings, the initial conditions take the form

$$
\begin{aligned}
& E(0)=E_{0}, \quad V(0)=V_{0}, \quad A(0)=1, \quad B(0)=B_{0}, \quad C(0)=C_{0}, \\
& H(0)=1, \quad \bar{T}_{r r}(0)=\bar{T}_{r r 0} .
\end{aligned}
$$

To facilitate comparison with the Newtonian solution (2.27) and (2.29) it is also convenient for us to define an initial time-offset parameter in terms of the reciprocal of the initial stretch rate,

$$
\tau_{0}=\frac{1}{E_{0}}>0
$$




\subsection{Exact solution for filament thickness and stretch rate}

We begin by finding a closed-form solution for $A(\tau), E(\tau)$ and $H(\tau)$ which are decoupled from the equations for $V, B$ and $C$ in (3.2). The solution of $(3.2 c)$ subject to the initial condition $A(0)=1$ is

$$
A(\tau)=e^{-\tau}
$$

Subsequently, the equation for the stretch rate $(3.2 a)$ reduces to a Riccati equation $[24,45]$

$$
\frac{d E}{d \tau}=-E^{2}+\frac{1}{4} \delta^{2} e^{-\tau}
$$

Using the standard change of variables $E(\tau)=W^{\prime}(\tau) / W(\tau)$ [24], (3.9) can be transformed into the second order ODE:

$$
\frac{d^{2} W}{d \tau^{2}}-\frac{1}{4} \delta^{2} e^{-\tau} W=0
$$

Another change of variables, $\zeta=\delta e^{-\tau / 2}$ transforms (3.10) into

$$
\zeta^{2} \frac{d^{2} \hat{W}}{d \zeta^{2}}+\zeta \frac{d \hat{W}}{d \zeta}-\zeta^{2} \hat{W}=0
$$

which is a modified Bessel equation of zero order $[24,1]$ with the general solution $\hat{W}(\zeta)=d_{1} I_{0}(\zeta)+d_{2} K_{0}(\zeta)$, where $I_{0}$ and $K_{0}$ are the modified Bessel functions of zero order and $d_{1}$ and $d_{2}$ are arbitrary constants. Consequently, we can write $E=(\zeta)$ in closed form as

$$
\hat{E}=(\zeta)=-\frac{\zeta}{2}\left(\frac{\alpha I_{1}(\zeta)-K_{1}(\zeta)}{\alpha I_{0}(\zeta)+K_{0}(\zeta)}\right)
$$

where $I_{1}$ and $K_{1}$ are the modified Bessel functions of first order. Then, from $E(\tau)=$ $\hat{E}\left(\delta e^{-\tau / 2}\right)$, the exact solution for the stretch rate is given by

$$
E(\tau)=-\frac{\delta e^{-\tau / 2}}{2}\left(\frac{\alpha I_{1}\left(\delta e^{-\tau / 2}\right)-K_{1}\left(\delta e^{-\tau / 2}\right)}{\alpha I_{0}\left(\delta e^{-\tau / 2}\right)+K_{0}\left(\delta e^{-\tau / 2}\right)}\right),
$$

where the constant $\alpha$ is chosen to satisfy the initial condition $E(0)=E_{0}$ and is given by

$$
\alpha=\frac{\delta K_{1}(\delta)-2 E_{0} K_{0}(\delta)}{\delta I_{1}(\delta)+2 E_{0} I_{0}(\delta)} .
$$

Further, using the kinematic condition $(3.2 \mathrm{~g})$ we can obtain the closed form solution for the filament radius,

$$
H(\tau)=\left(\frac{\alpha I_{0}(\delta)+K_{0}(\delta)}{\alpha I_{0}\left(\delta e^{-\tau / 2}\right)+K_{0}\left(\delta e^{-\tau / 2}\right)}\right)^{1 / 2},
$$

with $H(0)=1$. In $\S 4$, we compare this prediction for the time-dependence of the filament interface to measurements of the radius of a thinning filament drawn out by a falling drop. Equation (3.15) also lets us write the Hencky strain, the integral of the stretch rate 
[5], as

$$
\int_{0}^{\tau} E(s) d s=-2 \ln H(\tau)=\ln \left(\frac{\alpha I_{0}\left(\delta e^{-\tau / 2}\right)+K_{0}\left(\delta e^{-\tau / 2}\right)}{\alpha I_{0}(\delta)+K_{0}(\delta)}\right) .
$$

The functional forms of the stretch rate and filament radius for the UCM and OldroydB models are determined by a balance of inertial and elastic effects (see (3.2 $a$ ) and $(3.2 \mathrm{~g})$ ). This is different than other analyses that consider a balance between capillary and elastic forces which lead to exponential filament thinning and a constant stretch rate $[16,8,10]$.

In principle, given the exact solutions $(3.8,3.13,3.15)$, one could attempt to directly solve the remaining equations for $V, B, C$, and $\bar{T}_{r r}$. However, given the somewhat complicated form of (3.13), we do not seek the remaining closed-form analytic solutions. Instead of computing numerical solutions, we will re-examine the system (3.2) and the partial exact solution in different limiting regimes to better understand its structure and the qualitative dynamical behaviors that occur for viscoelastic fluid filaments. Of particular interest will be the comparison of the dynamics of viscoelastic versus Newtonian fluid filaments. For this comparison, we re-write the Newtonian solution from $\S 2.4$ in non-dimensional form using the scalings (3.1),

$$
\begin{gathered}
E_{\text {Newt }}(\tau)=\frac{1}{\tau+\tau_{0}}, \quad V_{\text {Newt }}(\tau)=-\frac{1}{2}\left(\tau+\tau_{0}\right)+\frac{Z_{0}}{\tau+\tau_{0}}, \\
A_{\text {Newt }} \equiv 0, \quad B_{\text {Newt }} \equiv 0, \quad C_{\text {Newt }}(\tau)=\frac{2}{\tau+\tau_{0}}, \\
H_{\text {Newt }}(\tau)=\frac{1}{\sqrt{1+\tau / \tau_{0}}},
\end{gathered}
$$

where the constants $\tau_{0}>0$ and $Z_{0}=V_{0} \tau_{0}+\frac{1}{2} \tau_{0}^{2}$ correspond to the initial conditions $(3.6 a)$, and the initial condition for the stress must satisfy $C_{0}=2 E_{0}>0$ in order to be consistent with (2.25).

In their dimensional forms, the Newtonian and viscoelastic solutions for the filament radius are independent of the fluid viscosity and surface tension; the viscoelastic solution does depend on the fluid elasticity and density through the parameter $\delta$. We show how these two solutions are related through limits of the Deborah number $\delta$ and by examining the dynamics for both long and short times.

\subsection{Long-time behavior}

As briefly described at the opening of $\S 3$, for long times the solutions of the filament equations converge to the Newtonian solution if the elastic effects are weak. We will now show that this is the case for all values of $\delta$.

To see that solutions of (3.2) converge to (3.17) as $\tau \rightarrow \infty$, consider the evolution of infinitesimal linear perturbations on the leading order terms in $(3.17 a, b)$,

$E(\tau)=\frac{1}{\tau}+\epsilon \tilde{E}(\tau), \quad V(\tau)=-\frac{1}{2} \tau+\epsilon \tilde{V}(\tau), \quad A(\tau)=\epsilon \tilde{A}(\tau), \quad B(\tau)=\epsilon \tilde{B}(\tau), \quad C(\tau)=\frac{2}{\tau}+\epsilon \tilde{C}(\tau)$. 
For $\tau \rightarrow \infty$, these perturbations satisfy the linearized version of (3.2),

$$
\begin{array}{r}
\frac{d \tilde{E}}{d \tau}=-\frac{2}{\tau} \tilde{E}+\frac{\delta^{2}}{4} \tilde{A}, \quad \frac{d \tilde{V}}{d \tau}=\frac{\tau}{2} \tilde{E}-\frac{1}{\tau} \tilde{V}+\tilde{B}, \\
\frac{d \tilde{A}}{d \tau}=-\tilde{A}, \quad \frac{d \tilde{B}}{d \tau}=-\left(1-\frac{1}{\tau}\right) \tilde{B}+\frac{\delta^{2}}{8} \tau \tilde{A}, \\
\frac{d \tilde{C}}{d \tau}=-\left(1-\frac{2}{\tau}\right) \tilde{C}+2\left(1+\frac{2-6 \omega}{\tau}\right) \tilde{E}+\frac{\omega \delta^{2}}{2} \tilde{A}+\frac{\psi}{2} \tau \tilde{B} .
\end{array}
$$

To leading order for $\tau \rightarrow \infty$, the general solution of this system is

$$
\left(\begin{array}{c}
\tilde{A} \\
\tilde{B} \\
\tilde{C} \\
\tilde{E} \\
\tilde{V}
\end{array}\right) \sim\left[d_{A}\left(\begin{array}{c}
1 \\
\frac{1}{8} \delta^{2} \tau^{2} \\
\frac{1}{32} \psi \delta^{2} \tau^{4} \\
-\frac{1}{4} \delta^{2} \\
-\frac{1}{8} \delta^{2} \tau^{2}
\end{array}\right)+d_{B}\left(\begin{array}{c}
0 \\
\tau \\
\frac{1}{2} \psi \tau^{3} \\
0 \\
-\tau
\end{array}\right)+d_{C}\left(\begin{array}{c}
0 \\
0 \\
\tau^{2} \\
0 \\
0
\end{array}\right)\right] e^{-\tau}+d_{E}\left(\begin{array}{c}
0 \\
0 \\
2 \tau^{-2} \\
\tau^{-2} \\
\frac{1}{2}
\end{array}\right)+d_{V}\left(\begin{array}{c}
0 \\
0 \\
0 \\
0 \\
\tau^{-1}
\end{array}\right),
$$

where the $d$-coefficients are constants corresponding to independent conditions for each of the perturbation modes. Notice that there are no growing perturbation modes; the first three modes have exponential decay. In fact all of the perturbation modes have sub-dominant contributions relative to the leading order Newtonian terms (3.18).

Consequently, linearized analysis suggests that all solutions of (3.2) converge to (3.17) as $\tau \rightarrow \infty$ for all $\delta$. It is notable that while the time scale ratio $\omega$ appears in (3.19), the leading order solution for $\tau \rightarrow \infty$ is independent of $\omega$. Recalling that for $\omega=0$ the Oldroyd-B equations (2.42) reduce to the UCM equations $(2.36,2.38)$, we can conclude that the influence of the retardation timescale is a higher order effect [50] and the relative difference between the UCM and Oldroyd-B models of filaments is negligible for long times.

The last two modes in (3.20), due to perturbations in the stretch rate and velocity, have algebraic decay for $\tau \rightarrow \infty$ and yield next-order contributions to $C, E, V$ in (3.18). These are not "true" instabilities; they correspond to infinitesimal perturbations within the continuous family of Newtonian solutions $(3.17 a, b)$, parametrized by $\tau_{0}, Z_{0}$. In particular for $d_{E}=-\tau_{\infty} / \epsilon$, where $\tau_{\infty}$ is a constant and $d_{V}=Z_{\infty} / \epsilon$ for some $Z_{\infty}$, equations (3.18) correspond to expansions for $\tau \rightarrow \infty$ of

$$
E(\tau) \sim \frac{1}{\tau+\tau_{\infty}}, \quad C(\tau) \sim \frac{2}{\tau+\tau_{\infty}}, \quad V(\tau) \sim-\frac{1}{2}\left(\tau+\tau_{\infty}\right)+\frac{Z_{\infty}}{\tau+\tau_{\infty}} .
$$

In comparing (3.21) with the corresponding solutions in (3.17), we note the difference is that the constants describing the initial conditions $\tau_{0}, Z_{0}$ have been replaced by other constants, $\tau_{\infty}, Z_{\infty}$. That is, with respect to the long-time behavior of the solution, the cumulative influence of the viscoelastic effects on the underlying Newtonian solution is to modify the effective initial conditions. Such effects are common in the long time asymptotic behavior of many mathematical systems with continuous families of attracting solutions, for example the calculation of optimal similarity solutions for diffusion equations [52]. In general, it may not be possible to find a "connection formula" between the initial 
data, parameterized by $\tau_{0}, Z_{0}$ and the long-time asymptotics, given by $\tau_{\infty}, Z_{\infty}$. However, the existence of the exact solution (3.13) makes this process straightforward for this problem.

\subsection{The weakly-viscoelastic limit}

For $\delta \rightarrow 0$, we show that the viscoelastic solution of (3.2) is comparable to a Newtonian solution of the form (3.21) for all times. We call this the weakly-viscoelastic limit.

From $(3.21)_{1}$, we observe that $\tau_{\infty}$ can be obtained from the limit

$$
\tau_{\infty}=\lim _{\tau \rightarrow \infty}\left(\frac{1}{E(\tau)}-\tau\right) .
$$

Using (3.13) for $E(\tau)$ yields an explicit "connection formula" $\tau_{\infty}=\tau_{\infty}\left(\tau_{0}, \delta\right)$. This expression is a rather complicated combination of Bessel functions, but for the moment we only consider its expansion in the limit $\delta \rightarrow 0$ with a finite, positive initial stretch rate, $\tau_{0}=E_{0}^{-1}=O(1)$, corresponding to the limit of weak viscoelastic effects,

$$
\tau_{\infty}=\tau_{0}-\left[\frac{1}{2}+\frac{\tau_{0}}{2}+\frac{\tau_{0}^{2}}{4}\right] \delta^{2}+\left[\frac{5}{64}+\frac{5 \tau_{0}}{32}+\frac{5 \tau_{0}^{2}}{32}+\frac{\tau_{0}^{3}}{16}\right] \delta^{4}-O\left(\delta^{6}\right) .
$$

We note that for $\delta=0$ we recover $\tau_{\infty}=\tau_{0}$, that is, the value corresponding to the initial stretch rate for the Newtonian solution $(3.17 a)_{1}$. For $\delta \ll 1$, (3.23) implies that $\tau_{\infty}$ is less than $\tau_{0}$ and is a decreasing function of $\delta$. The implication of this result is that very weak viscoelastic effects yield an apparent increase in the initial stretch rate $E(0)=E_{\infty}$, for $\delta \rightarrow 0$

$$
E_{\infty} \equiv \frac{1}{\tau_{\infty}} \sim E_{0}+\frac{1+2 E_{0}+2 E_{0}^{2}}{4} \delta^{2}+\frac{6+22 E_{0}+27 E_{0}^{2}+16 E_{0}^{3}}{64} \delta^{4},
$$

relative to that of a Newtonian filament.

Using comparison theorems for first order ODEs, the Newtonian solutions based on $\tau_{\infty}, \tau_{0}$ yield upper and lower bounds on the stretch rate $E(\tau)$ for all times (see Figure 4),

$$
\frac{1}{\tau+\tau_{0}} \leqslant E(\tau) \leqslant \frac{1}{\tau+\tau_{\infty}}
$$

A direct consequence of $(3.21)_{1}$ used in the kinematic condition $(3.2 \mathrm{~g})$ is that the long-time evolution of the filament thickness also follows the Newtonian behavior for $\tau \rightarrow \infty$,

$$
H(\tau) \sim \frac{H_{\infty}}{\left|1+\tau / \tau_{\infty}\right|^{1 / 2}},
$$

where the constant $H_{\infty}$ gives the expected initial filament thickness at $\tau=0$ based on the long-time asymptotics for a comparable Newtonian fluid filament. We have written (3.26) in a slightly different form than $(3.17 c)$ in order to ensure that the filament thickness is always real-valued for all values of $\tau_{\infty}$; we will see that $\tau_{\infty}<0$ can occur in the next section. The parameter $\tau_{\infty}$ has a directly observable physical interpretation for the dynamics of the filament. That is, $\left|\tau_{\infty}\right|$ represents a time-delay before the thinning of the 


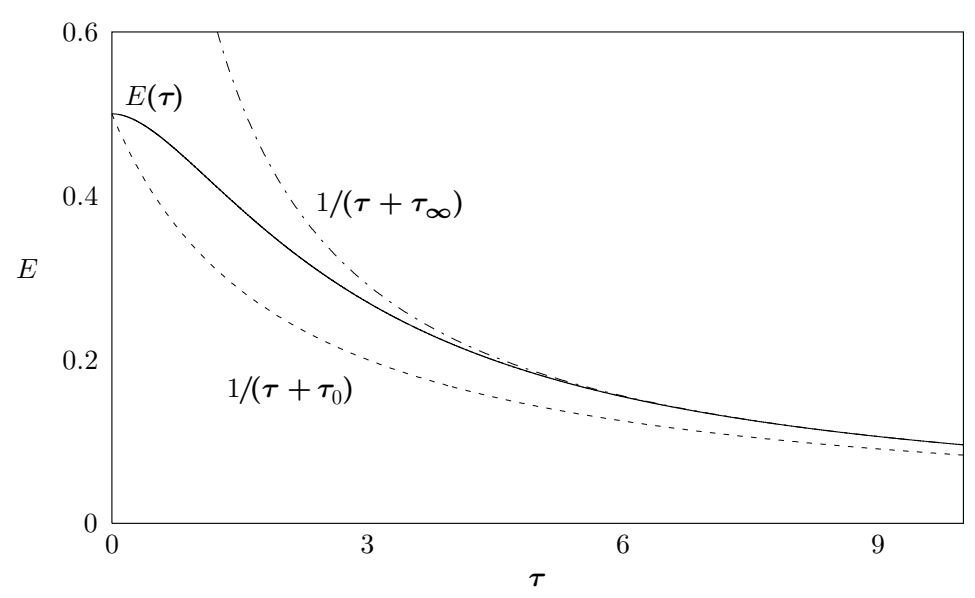

FIGURE 4. The stretch rate $E(\tau)$ from (3.13) (solid) and the Newtonian upper/lower bounds based on $\tau_{\infty}$ (dot-dashed) and $\tau_{0}$ (dashed) in the weakly-viscoelastic case with $\delta=1 / 2$, see (3.25).

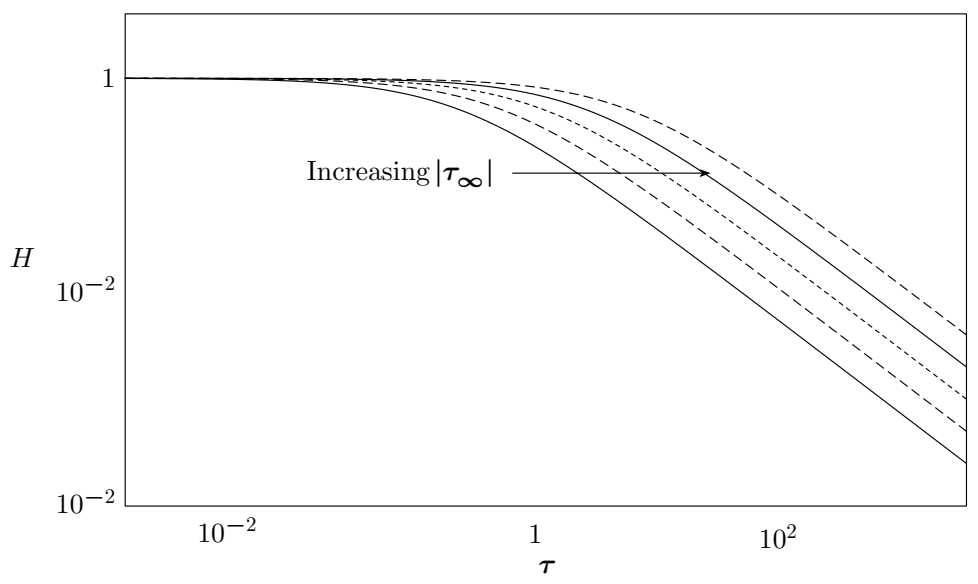

FIGURE 5. Delay of the onset of the asymptotic power-law thinning behavior of $H(\tau)$ with increasing $\left|\tau_{\infty}\right|$ with $\tau_{\infty}>0$ for the weakly-viscoelastic case.

filament approaches a scaling law, $H=O\left(\tau^{-1 / 2}\right)$ for $\tau \gg\left|\tau_{\infty}\right|$. This behavior is most clearly exhibited on a log-log graph, as shown in Figure 5.

In general, the value of the $H_{\infty}$ parameter can be obtained from the limit

$$
H_{\infty}=\lim _{\tau \rightarrow \infty} H(\tau) \sqrt{\frac{\tau}{\left|\tau_{\infty}\right|}} .
$$

Using (3.22) for $\tau_{\infty}$ and the exact solution (3.15) for $H(\tau)$, we obtain $H_{\infty}$ in terms of $\delta$ and $\tau_{0}$. Expanding the resulting expression for $\delta \rightarrow 0$ and $\tau_{0}=O(1)$ yields

$$
H_{\infty}=1+\left(\frac{2+\tau_{0}}{8 \tau_{0}}\right) \delta^{2}+\left(\frac{8 \tau_{0}^{2}+15 \tau_{0}+12}{128 \tau_{0}^{2}}\right) \delta^{4}+O\left(\delta^{6}\right) .
$$




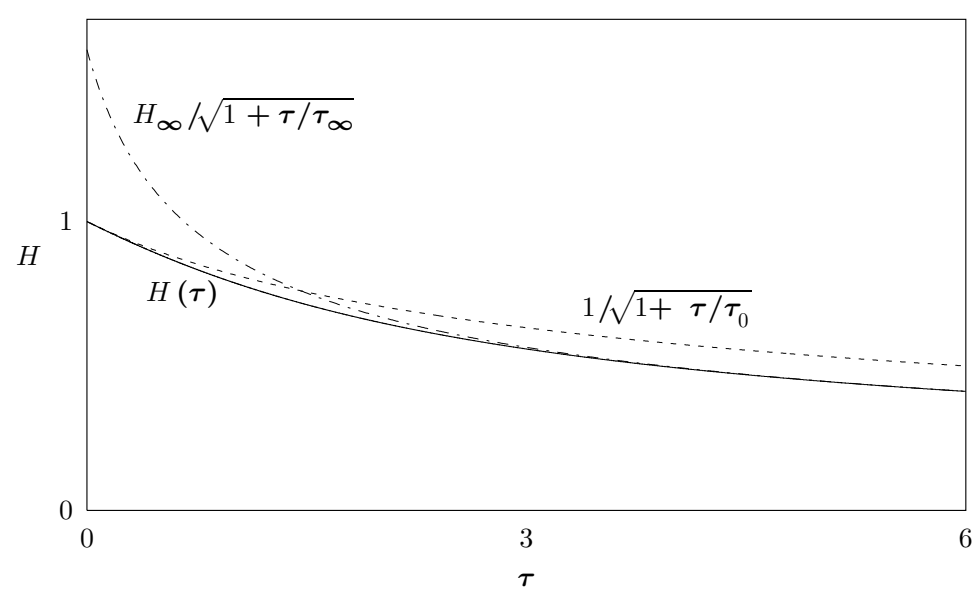

FIGURE 6. $H(\tau)$ (solid) and the Newtonian estimates based on $\tau_{\infty}$ (dot-dashed) and $\tau_{0}$ (dashed) in the weakly-viscoelastic case with $\delta=1 / 2$.

Using $\tau_{0}=1 / E_{0}$, this result can be written in terms of the initial stretch rate in the general form

$$
H_{\infty} \sim \sum_{n=0} P_{n}\left(E_{0}\right) \delta^{2 n} \quad \delta \rightarrow 0
$$

where the $P_{n}\left(E_{0}\right)$ are $n$-th order polynomials in $E_{0}$ with all positive coefficients. This result suggests that the influence of viscoelastic effects in the weakly-viscoelastic limit is to yield an apparent increase in the filament thickness relative to that expected from a Newtonian filament, $H_{\infty} \geqslant 1$. From (3.25) and comparison theorems applied to $(3.2 \mathrm{~g})$, it can be shown that (3.26) and $(3.17 c)$ are both upper bounds for $H(\tau)$ for all time, with $(3.17 c)$ giving a tighter bound for short times and $H(\tau)$ converging to (3.26) for long times (see Figure 6).

\subsection{Transient behavior and the strongly-viscoelasticlimit}

Having established that the long-time dynamics approaches Newtonian behavior, we examine what influence the viscoelastic models can yield for short to moderate times. Here we explore the possible qualitative changes from Newtonian behavior that can occur due to transient viscoelastic effects. For the weakly-viscoelastic case, with $\delta \rightarrow 0$, we showed that such transient effects produced small changes to the initial conditions for $E(0)$ (3.24) and $H(0)$ (3.28). Now, we will describe the strongly-viscoelastic regime, where the transient effects would have to come from unphysical initial conditions.

For a Newtonian filament the stretch rate is monotone decreasing $(3.17 a)_{1}$ for all times. In contrast, for viscoelastic filaments, we see from (3.9) that for Deborah numbers that are large compared to the initial stretch rate, $E(\tau)$ will initially be increasing if

$$
E_{0}<\frac{\delta}{2}
$$

More generally from (3.9), $E(\tau)$ will be increasing so long as $E(\tau)<\frac{1}{2} \delta e^{-\tau / 2}$. In terms 
of $\zeta=\delta e^{-\tau / 2}$, the time $\tau_{\max }$ when the maximum of $E(\tau)$ occurs can be found by solving the transcendental equation $\hat{E}\left(\zeta_{\max }\right)=\zeta_{\max } / 2$, or equivalently in terms of $\left(\delta, E_{0}\right)$ using (3.12),

$$
K_{1}\left(\zeta_{\max }\right)-\alpha I_{1}\left(\zeta_{\max }\right)=K_{0}\left(\zeta_{\max }\right)+\alpha I_{0}\left(\zeta_{\max }\right) .
$$

Instead of pursuing a direct solution of (3.31), to gain further insight into the shorttime behavior we consider a perturbation expansion of the solution of (3.9) in the limit suggested by (3.30), that is, for sufficiently large $\delta$.

Let $\delta \rightarrow \infty$ with $E=\delta \mathscr{E}$, then (3.9) becomes the singularly perturbed equation

$$
\frac{1}{\delta} \frac{d \mathscr{E}}{d \tau}=-\mathscr{E}^{2}+\frac{1}{4} e^{-\tau}
$$

where $\mathscr{E}(0)<1 / 2$ to satisfy (3.30). Consequently, we can obtain an expansion of (3.13) valid for $O(1)$ times by solving a series of algebraic problems for the outer solution of (3.32) as $\delta \rightarrow \infty$ with $\mathscr{E}(\tau)=\mathscr{E}_{0}(\tau)+\delta^{-1} \mathscr{E}_{1}(\tau)+\delta^{-2} \mathscr{E}_{2}(\tau)+\cdots$. This yields the stretch rate as

$$
E(\tau) \sim \frac{\delta}{2} e^{-\tau / 2}+\frac{1}{4}-\frac{\delta^{-1}}{16} e^{\tau / 2}+\frac{\delta^{-2}}{16} e^{\tau} \quad \delta \rightarrow \infty .
$$

Notice that the maximum stretch rate is $O(\delta)$ and that this intermediate behavior is independent of the initial conditions. This solution is equivalent to an expansion of the exact solution (3.12) for $\zeta \rightarrow \infty$. In terms of $\tau$, (3.33) is valid for $0<\tau \ll O(\ln \delta)$, for longer times, there is a transition to Newtonian behavior, $(3.21)_{1}$.

For shorter times, to connect to the initial conditions, we rescale time by $\tau=\mathscr{T} / \delta$ in (3.32) to obtain an inner problem for the initial layer as $\delta \rightarrow \infty$,

$$
\frac{d \mathscr{E}}{d \mathscr{T}}+\mathscr{E}^{2}=\frac{1}{4} e^{-\mathscr{T} / \delta} \sim \frac{1}{4}\left(1-\frac{1}{\delta} \mathscr{T}+\frac{1}{2 \delta^{2}} \mathscr{T}^{2}+O\left(\delta^{-3}\right)\right) .
$$

Writing a regular perturbation expansion for the solution of this equation, $\mathscr{E}(\mathscr{T})=\mathscr{E}_{0}(\mathscr{T})+$ $\delta^{-1} \mathscr{E}_{1}(\mathscr{T})+\cdots$, we obtain the leading order inner solution, valid on $0 \leqslant \tau \ll O\left(\delta^{-1}\right)$ as

$$
E(\tau) \sim \frac{\delta}{2}\left(\frac{\delta+2 E_{0}-\left(\delta-2 E_{0}\right) e^{-\delta \tau}}{\delta+2 E_{0}+\left(\delta-2 E_{0}\right) e^{-\delta \tau}}\right) .
$$

By examining the forms of the initial layer (3.35) and the intermediate time solution (3.33), we conclude that $\tau_{\max }=O\left(\delta^{-1}\right)$ and $E_{\max } \lesssim \delta / 2$, for large $\delta$, as shown in Figure 7 .

In turning our focus to behavior for large $\delta$, we note that the expansions for $\tau_{\infty}(3.23)$ and $H_{\infty}$ (3.28) for $\delta \rightarrow 0$ are not valid for $\delta \rightarrow \infty$. From (3.23) we see that when $\tau_{0}=\delta^{2} / 2$ that $\tau_{\infty} \approx 0$ suggesting an unphysical infinite initial stretch rate, $E_{\infty}=1 / \tau_{\infty}$. Hence, we re-examine the definitions of $\tau_{\infty}$ (3.22) and $H_{\infty}$ (3.27) in the strongly-viscoelastic limit, for large $\delta$. Figure 8 shows $\tau_{\infty}$ and $H_{\infty}$ for a fixed value of the initial stretch rate, $E_{0}=1$, over a finite range of values for $\delta$. Observe that for small values of $\delta$, below a certain critical value $\delta_{*}$, we obtain results consistent with the expansions for the effective initial conditions in the previous section, that is $\tau_{\infty}>0$ and $H_{\infty}>1$. This is the weakly-viscoelastic or "nearlyNewtonian" regime. As $\delta \rightarrow \delta_{*}, \tau_{\infty} \rightarrow 0$ and $H_{\infty}$ is singular of the form $O\left(\left|\delta-\delta_{*}\right|^{-1 / 2}\right)$. For $\delta>\delta_{*}$, having a negative value for $\tau_{\infty}$ means that (3.21) and (3.26) become singular 


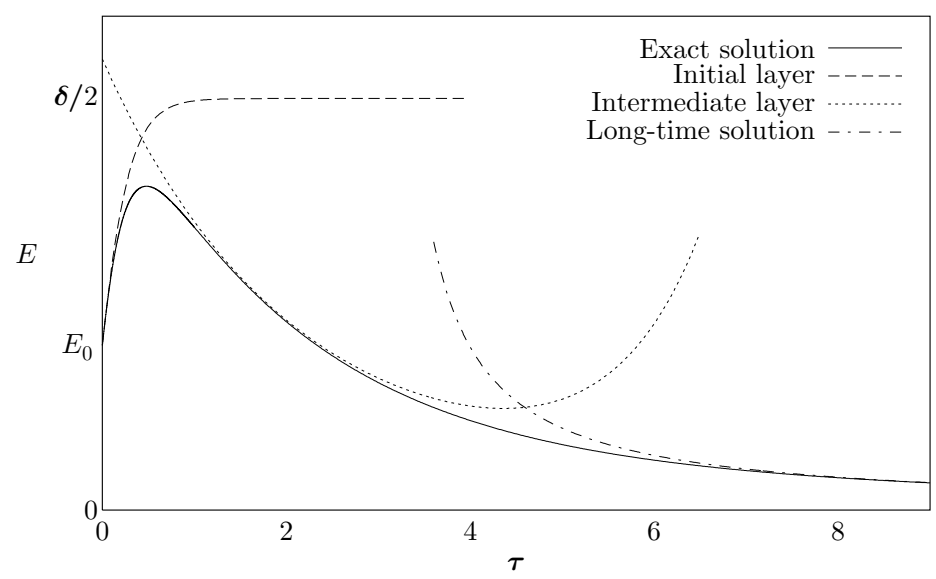

FIGURE 7. The asymptotic structure of $E(\tau)$ (3.13) via short (3.35), intermediate (3.33), and long-time $(3.21)_{1}\left(\right.$ with $\left.\tau_{\infty}<0\right)$ asymptotic solutions for the strongly-viscoelastic case with $\delta=5$.
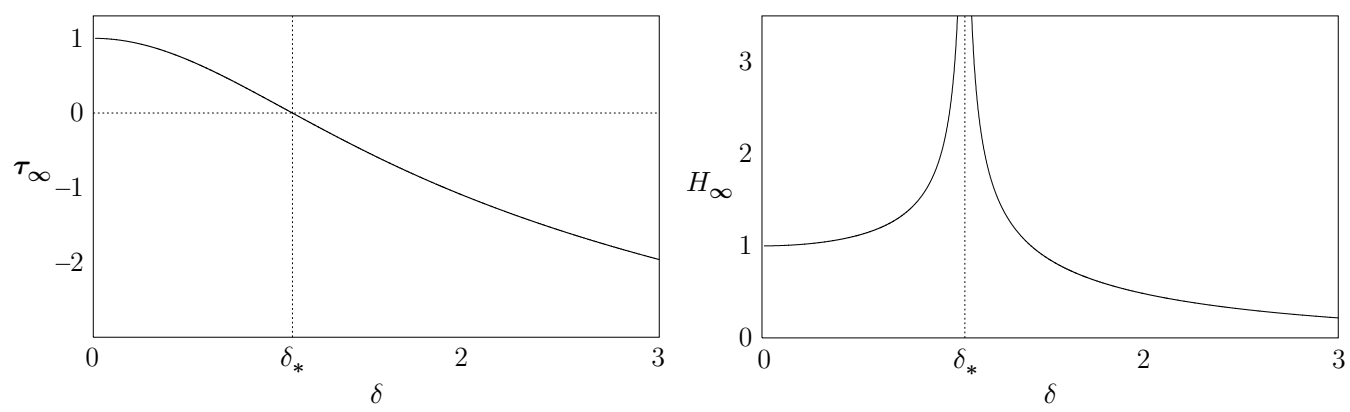

FIGURE 8. For fixed $E_{0}=1, \tau_{\infty}$ (left) and $H_{\infty}$ (right) plotted as a function of the Deborah number $\delta$ with $\delta_{*} \approx 1.056$ for $E_{0}=1$ separating weakly- and strongly-viscoelastic regimes.

at some finite positive time (at $\tau=\left|\tau_{\infty}\right|$ ). This behavior is unphysical and does not occur in solutions of the viscoelastic model equations (3.2). This suggests that the long-time asymptotic behaviors given by (3.21) and (3.26) can only be applied for times $\tau>-\tau_{\infty}$. For earlier times, the transient dynamics of the viscoelastic filament are not comparable to any Newtonian behavior. This is in contrast with the weakly-viscoelastic case, where the solution of (3.2) could be compared with the Newtonian behavior given by (3.21) for all times $\tau \geqslant 0$. Consequently, we call the case with $\tau_{\infty}<0$ the strongly-viscoelastic case.

We define the borderline between the weakly- and strongly-viscoelastic cases in terms of the condition that the apparent initial stretch rate $E_{\infty}$ diverges, or equivalently that $\tau_{\infty}\left(E_{0}, \delta\right)=0$. This condition defines the critical initial stretch rate $E_{0}=E_{0}^{*}(\delta)$. From (3.23) with $\tau_{0}=1 / E_{0}$, we find that for $\delta \rightarrow 0$,

$$
E_{0}^{*}(\delta) \sim \frac{2}{\delta^{2}}
$$

For large $\delta$ this suggests that the critical $E_{0}$ is small; to test the limits of validity of this criterion, we examine the limit of $\tau_{\infty}$ (3.22) for $E_{0} \rightarrow 0$ (equivalently for $\tau_{0} \rightarrow \infty$ ). Enforcing 


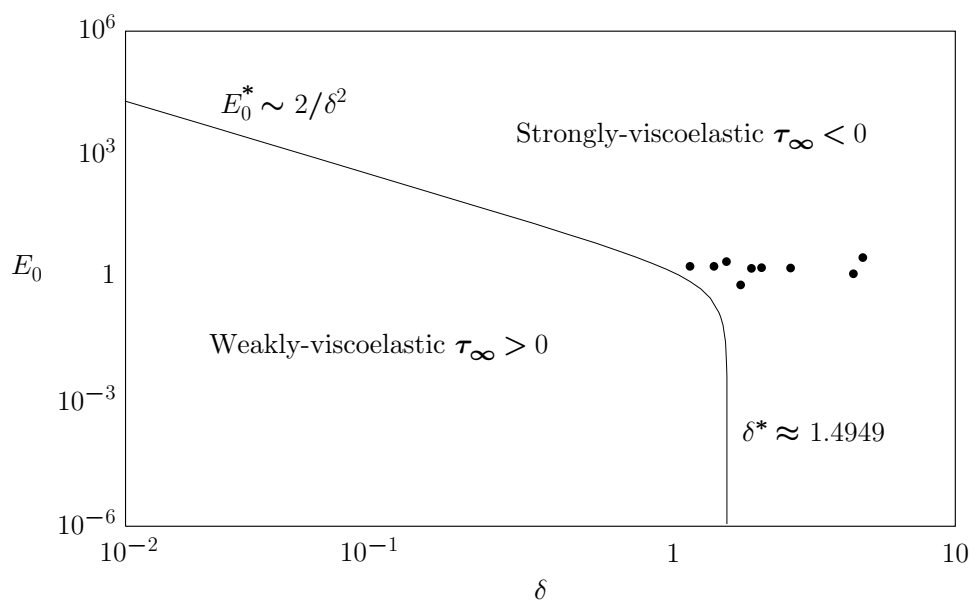

FIGURE 9. The dividing line between the weakly- and strongly-viscoelastic regimes. The solid dots correspond to conditions for the experiments described in $\S 4$.

$\tau_{\infty}=0$ to leading order as $\tau_{0} \rightarrow \infty$ yields the equation for $\delta$,

$$
\ln \left(\frac{2}{\delta}\right)-\gamma+\frac{K_{1}(\delta)}{I_{1}(\delta)}=0,
$$

where $\gamma \approx 0.577215$ is the Euler-Mascheroni constant. This equation has a single real root for $\delta$, which must be obtained numerically,

$$
\delta^{*} \approx 1.494894878 \cdots
$$

There are no solutions of $\tau_{\infty}=0$ for $\delta>\delta^{*}$. The conclusion is that for $\delta>\delta^{*}$ all filament dynamics are strongly-viscoelastic, for any $E_{0}$ (see Figure 9); for $\delta<\delta^{*}$ a solution of (3.2) can fall in either regime, depending on its initial value for the stretch rate. For increasing $\delta,(3.27)$ yields an exponential thinning of the effective filament thickness,

$$
H_{\infty} \sim\left(\frac{2 \pi}{\delta \ln ^{2} \delta}\right)^{1 / 4} e^{-\delta / 2}, \quad \delta \rightarrow \infty .
$$

While $H_{\infty}$ depends on $E_{0}$ only in higher-order terms as $\delta \rightarrow \infty$, for cases of practical interest, with $\delta=O(1)$, these terms may be needed to obtain sufficiently accurate values of (3.27).

Apart from the applicability-in-time of the Newtonian estimate (3.21), the importance of this dividing line between the weakly- and strongly-viscoelastic regimes is the qualitative differences that accompany it. For weakly-viscoelastic cases, as $\delta$ increases, $\left|\tau_{\infty}\right|$ decreases (see Figure 8(a)), consequently Figure 5 suggests that increasing viscoelastic effects actually decreases the time-delay before power-law thinning occurs. In contrast, for the stronglyviscoelastic case, as $\delta$ increases, then $\left|\tau_{\infty}\right|$ increases (see Figure 8(a)) and the time-delay increases with viscoelastic effects - this is the behavior that is more expected. Delayed filament thinning has been observed experimentally by Amarouchene et al. [2] and by 
Anna \& McKinley [3]. In experiments with drops, Amarouchene et al. [2] found the delay in the thinning filament increased for higher polymer concentration and higher molecular weight, corresponding to an increase in the fluid elasticity. At least in terms of this property of the solutions, the $\tau_{\infty}=0$ criterion serves to separate qualitatively opposite trends between nearly-Newtonian and strongly-viscoelastic fluid filaments.

\subsection{Effects of viscoelasticity on the stress}

Here we examine the effects of viscoelasticity on the stress, and highlight differences between the UCM and Oldroyd-B models. To isolate viscoelastic effects, we consider the trace of the stress tensor, $\operatorname{tr}(\mathbf{T})=T_{z z}+2 T_{r r}$, which using the expansion for the axial stress (2.24), can be written as

$$
\operatorname{tr}(\mathbf{T}(z, t))=\frac{1}{2} a z^{2}+b z+c+2 T_{r r}
$$

For a Newtonian filament, this reduces to $\operatorname{tr}(\mathbf{T})=0+0+2 \eta \dot{\varepsilon}+2(-\eta \dot{\varepsilon}) \equiv 0$. In fact, (3.40) is identically zero for all incompressible Newtonian flows, since for Newtonian fluids the stress is proportional to $\mathbf{D}(2.25)$ and the trace of $\mathbf{D}$ yields the continuity equation (2.2). Consequently, (3.40) gives a measure of the "excess stress" due to viscoelasticity. Nondimensionalizing (3.40) by $\Sigma=\lambda \operatorname{tr}(\mathbf{T}) / \eta$ and $Z=z /\left(g \lambda^{2}\right)$ we obtain

$$
\Sigma(Z, \tau)=\frac{\psi \delta^{2}}{8} A(\tau) Z^{2}+\psi B(\tau) Z+C(\tau)+2 \bar{T}_{r r}(\tau)
$$

To evaluate the excess stress, we expand $B, C$, and $\bar{T}_{r r}$ in the weakly-viscoelastic limit $\delta \rightarrow 0$. Given the coupled form of the viscoelastic model equations (3.2), we also expand the stretch rate using (3.13) and $V(\tau)$. As $\delta \rightarrow 0$, we obtain

$$
\begin{aligned}
E(\tau) \sim & \frac{1}{\tau+\tau_{0}}+\delta^{2}\left(\frac{1+\left(1+\tau_{0}\right)^{2}}{4\left(\tau+\tau_{0}\right)^{2}}-\left[\frac{1}{4}+\frac{1}{2\left(\tau+\tau_{0}\right)}+\frac{1}{2\left(\tau+\tau_{0}\right)^{2}}\right] e^{-\tau}\right)+O\left(\delta^{4}\right), \quad(3.42 a) \\
V(\tau) \sim & \frac{V_{0} \tau_{0}}{\tau+\tau_{0}}-\frac{\tau+\tau_{0}}{2}+\frac{1}{2} \frac{\tau_{0}^{2}}{\tau+\tau_{0}}+\frac{B_{0}}{\tau+\tau_{0}}\left(\frac{\tau_{0}^{2}+2 \tau_{0}+2}{\tau_{0}}\right) \\
& -\frac{B_{0}}{\tau+\tau_{0}}\left(\frac{\tau^{2}+2 \tau+2}{\tau_{0}}+2 \tau+2+\tau_{0}\right) \mathrm{e}^{-\tau}+O\left(\delta^{2}\right), \\
A(\tau)= & \mathrm{e}^{-\tau}, \quad B(\tau) \sim \frac{B_{0}\left(\tau+\tau_{0}\right)}{\tau_{0}} \mathrm{e}^{-\tau}+O\left(\delta^{2}\right), \\
C(\tau) \sim & {\left[\frac{2 \omega}{\tau+\tau_{0}}+(\omega-1)\left[1+\tau+\tau_{0}+\left(\tau+\tau_{0}\right)^{2} \mathrm{e}^{-\left(\tau+\tau_{0}\right)} \mathrm{E}_{1}\left(-\left(\tau+\tau_{0}\right)\right)\right]+O\left(\mathrm{e}^{-\tau}\right)\right]+O\left(\delta^{2}\right), } \\
\bar{T}_{r r}(\tau) \sim & -\frac{1}{\tau+\tau_{0}}+\frac{\left(1+\bar{T}_{r r 0} \tau_{0}\right)}{\tau+\tau_{0}} \mathrm{e}^{-\tau}+O\left(\delta^{2}\right),
\end{aligned}
$$

where $E_{1}$ is the exponential integral function [1], and we report only the algebraic terms of $C$. The only dependence on $\psi$, representing the influence of gravitational, elastic and viscous effects, in (3.42) occurs in exponentially small terms in $C$. The form of $C$ is 
complicated, however in the asymptotic limit $\tau \rightarrow \infty$, this expansion simplifies to

$$
C(\tau) \sim \frac{2}{\tau+\tau_{0}}+\frac{6(1-\omega)}{\left(\tau+\tau_{0}\right)^{2}} .
$$

Observe that to leading order, this agrees with the result for the Newtonian filament $C=2 E$. Note that all of the spatially dependent terms in the excess stress (3.41) decay exponentially in time. In the limit $\tau \rightarrow \infty$,

$$
\Sigma(\tau) \sim \frac{6(1-\omega)}{\left(\tau+\tau_{0}\right)^{2}}
$$

Assuming $0 \leqslant \omega \leqslant 1$ [31], the excess stress is largest when $\omega=0$, corresponding to the UCM model, and zero when $\omega=1$, corresponding to "Newtonian-like" behavior. Thus at long times, the effect of the additional solvent stress in the Oldroyd-B model $\left(\omega=\lambda_{r} / \lambda \neq 0\right.$ in (2.39)) is to decrease the effective viscoelastic stress. This behavior is controlled by the axial stress term $C(\tau)$.

Next considering intermediate times, we know the axial stress depends principally upon $C(\tau)$ after some transient period since $A$ and $B$ exponentially decay. Figure 10 shows a comparison of numerical solutions of $C(\tau)$ for the UCM $(\omega=0)$ (solid) and OldroydB (dashed) models using (3.2) where we see the UCM stress bounds from above the Oldroyd-B stress. This behavior can be understood by writing the evolution equation for $C(\tau)$ in the form,

$$
\frac{d C}{d \tau}=-(1-2 E) C+2 E-\psi B V+2 \omega\left(\frac{d E}{d \tau}-2 E^{2}\right) .
$$

In $\S 3.5$ it was shown that $E$ is a decreasing function after some transient period. Consequently after this transient period

$$
2 \omega\left(\frac{d E}{d \tau}-2 E^{2}\right)<0
$$

Therefore, given the same initial data, the stress from the UCM model $(\omega=0)$ is larger than the Oldroyd-B model $(\omega>0)$ after a transient period.

Figure 10 shows a typical solution where $C(\tau)$ attains a maximum before monotonically decreasing. In experiments, Sridhar et al. observed a similar transient increase then relaxational decay in the stress for polymer solutions in an elongational flow [48]. Asymptotic and numerical studies of a simple model for an extending viscoelastic filament attached to a mass by Keiller, show qualitatively similar buildup then decay of the elastic stress [27]. This behavior can be understood by considering the polymer's response to an extensional flow, as studied in numerical simulations by Hinch [23]. Initially, the molecules stretch due to sufficiently high strain rates, which results in a buildup in the axial stress. The peak in the stress occurs approximately at the time the molecules undergo a stretch-to-coil transition $\left(\tau \approx 1 / E_{\mathrm{tr}}=2\right.$ in Figure 10$)$, so that at longer times the molecules relax from their stretched state and the stress decays. In contrast, the axial stress of a Newtonian filament decays monotonically as $1 / \tau$ due to viscous effects. Thus, we attribute the initial 


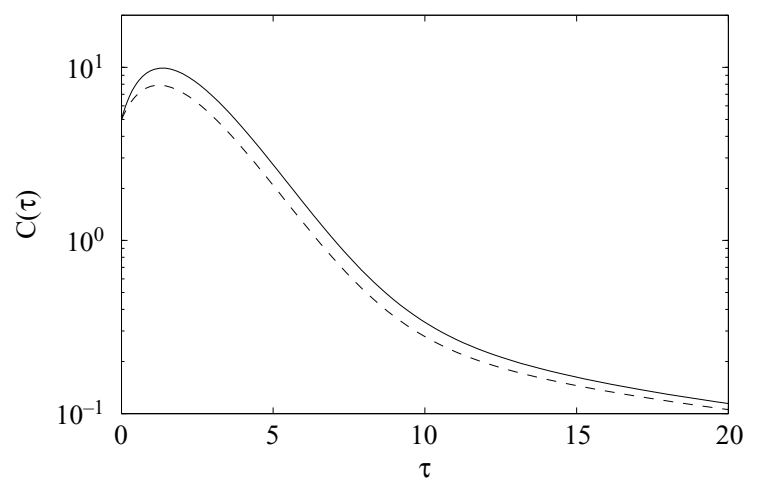

Figure 10. The axial stress coefficient $C(\tau)$ for the UCM $(\omega=0)$ (solid) and Oldroyd-B $(\omega=1 / 2)$ (dashed) models from numerical solution of (3.2) with $E_{0}=1, V_{0}=-1, B_{0}=1, C_{0}=5, \psi=1 / 2$, and $\delta=1 / 2$.

increase of the stress to elastic effects, and the decay of the stress to a "Newtonian-like" spatially uniform field to coiling of the polymer molecules with viscous effects dominant.

\section{Comparison with experiment}

When a drop falls from its source it draws out a filament that attaches it to the source. Examples are shown in Figure 1 for a Newtonian fluid, and in Figure 2 for a viscoelastic fluid. The dynamics of this filament, from its initial formation to pinch-off, depend strongly on the fluid properties.

In the experiment shown in Figure 1, the filament is nearly cylindrical over a brief time interval (between frames (a) and (c)). Eventually the filament loses its uniformity and tapers down at its connections to the drop and orifice, as shown in frame (c). Within a few milliseconds pinch-off occurs, first at the filament's connection to the drop (frame (e)), then at its connection to the orifice (frame (f)), and the free filament that remains collapses to form one satellite drop (frame (g)).

The fluid shown in Figure 2 is the same as that shown in Figure 1, with a small amount of polymer added. Given nearly the same experimental conditions, we observe strikingly different behavior. The Newtonian solvent drop pinches-off in tens of milliseconds, while the drop with the polymer solution takes over one second to pinch-off. The drop length at the time of pinch-off is also very different; $1.4 \mathrm{~cm}$ for the Newtonian solvent versus approximately $1 \mathrm{~m}$ for the polymer solution [44]. As in the Newtonian case, the viscoelastic filament is nearly cylindrical over some time period, between frames (a) and (d). After pinch-off of the main drop, the filament that remains sways, while elastically retracting back into the orifice, as shown in frames (d) and (e). It is the viscoelastic properties of the polymer fluid which account for these different and interesting dynamics [25, 2, 44].

Henderson et al. [22] considered the exact solution for a Newtonian stretching filament as a model for the thinning of the finite filament attached to a falling drop. In an experimental study with a series of (Newtonian) silicone oils, they showed the Newtonian solution (2.29) correctly predicted the temporal scaling of the thinning filament during the time when the filament is cylindrical [22]. Here we consider the exact solution for the 
filament radius $H(\tau)$ (3.15), derived for the UCM and Oldroyd-B constitutive equations, as a model for the thinning of the filament attached to a falling drop of viscoelastic fluid. We compare this exact solution to experimental measurements of the changing filament interface for several semi-dilute polymer solutions.

\subsection{Experimental fluids and apparatus}

We conducted nine experiments with solutions of the polymer xanthan gum [40] at two different concentrations $(0.078 \%$ and $0.156 \%$ by weight $)$, mixed in $80: 20$ glycerol/water with and without the salt, potassium chloride $\mathrm{KCl}(0-0.047 \%$ by weight $)$ [44]. The surface tension of these fluids was measured at room temperature using a Fisher model 21 tensiomat, and varied by $1 \%$ from $\gamma=67.4$ dyne $/ \mathrm{cm}$. The fluid density varied by less than $1 \%$ from $\rho=1.28 \mathrm{~g} / \mathrm{cm}^{3}$. Xanthan gum is a high molecular weight $\left(\approx 5 \times 10^{6}\right)$, water-soluble, anionic polysaccharide produced by the bacterium Xanthomonas Campestris [34]. The xanthan gum ${ }^{2}$ and $\mathrm{KCl}$ were gently mixed in distilled de-ionized water until the solution was homogeneous. Glycerol was next added, and the solution was mixed for up to 24 hours, and allowed to sit for another day before use.

Measurements of the shear rheology for these xanthan gum solutions indicate the relaxation time and shear viscosity increase with increasing polymer concentration, and decrease with increasing salt concentration [44, 45, 46]. These fluids are also known to shear-thin (the shear viscosity decreases with increasing shear rate) and strain-harden $[45,46,29,19]$. The zero-shear viscosity and relaxation time of $0.16 \%$ xanthan gum mixed in 80:20 glycerol/water (with no salt) at $25^{\circ} \mathrm{C}$ is $\eta_{0} \simeq 500 \mathrm{P}$ and $\lambda \simeq 40 \mathrm{~s}$ [46]; these values represent an upper bound for the fluids considered here.

The experimental apparatus that produced the drops was similar to those used previously [22]. The fluid flow was driven by gravity from a $32 \mathrm{~cm}^{3}$ plastic (Lucite) reservoir, which was open to the atmosphere, into a tube of length $2.5 \mathrm{~cm}$, through a needle valve, which controlled the flow rate, and finally through a lower tube of length $6.6 \mathrm{~cm}$ with inner and outer radii of 1.54 and $2.17 \mathrm{~mm}$. The $0.63-\mathrm{mm}$-wide edge of the orifice was machined flat to allow for a reproducible contact line at the orifice/fluid/air interface. In all of the experiments the period of the drops was about $30 \mathrm{~s} /$ drop. The experimental apparatus was set on a vibration isolated table and enclosed in a box to reduce air currents. Images and video sequences of the drops were obtained using a Kodak Ektapro 1012 EM Motion Analyzer; details of the illumination used in these experiments can be found in [22]. The spatial resolution of the images was $21 \mu \mathrm{m} /$ pixel and the temporal resolution in the experiments ranged between 1000-3000 images/s.

\subsection{Experimental results}

Here we compare the exact solution $H(\tau)$ (3.15) to the radius of the thinning filament attached to a falling drop. In the experiments, we measured the diameter, $2 h$, of the filament at a fixed $z$ location during the time before the drop pinched-off and while $\partial h / \partial z \approx 0$, that is, while the filament was cylindrical. This corresponds to the interval between frames (a)-(d) in Figure 2. Using a nonlinear curve-fitting program, we determine

2 Vanzan NF, provided by R. T. Vanderbilt Co. (Norwalk, CT). 

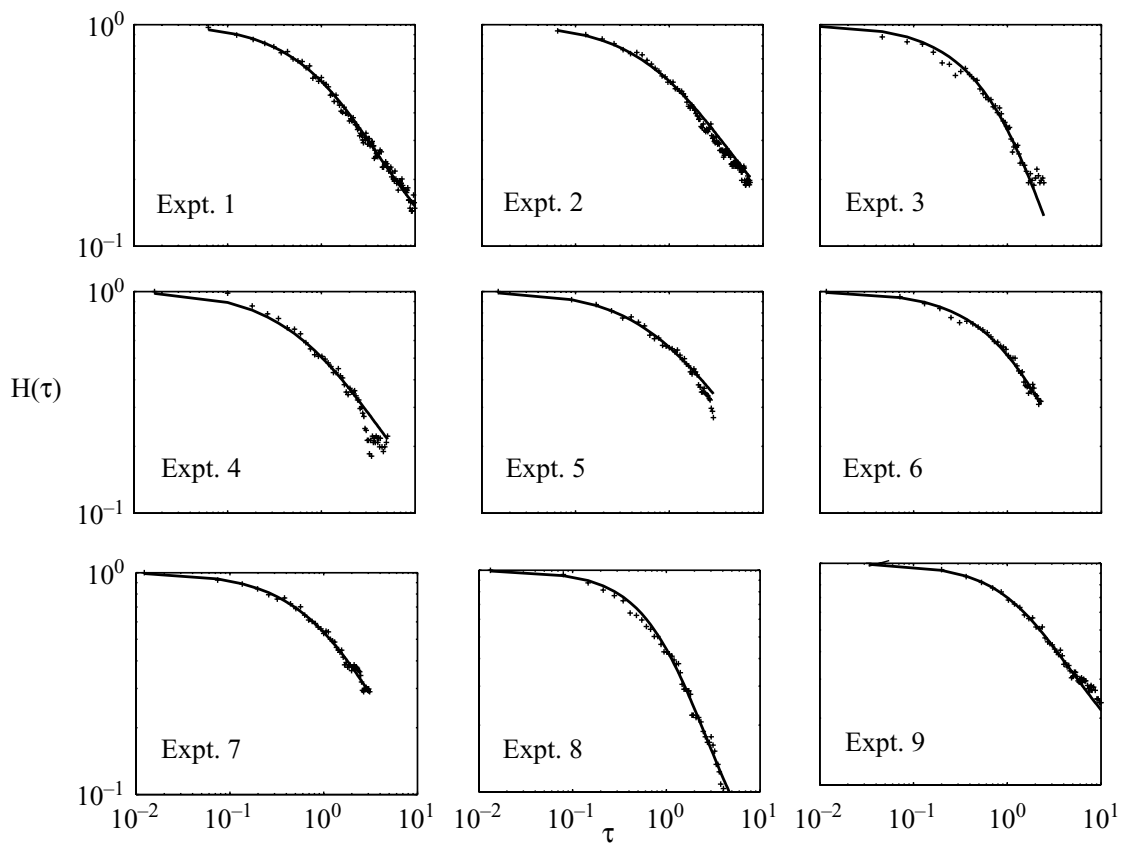

Figure 11. Comparison of the exact solution for the filament radius $H(\tau)$ (solid line) with measurements (pluses) for: $0.078 \%$ xanthan gum (x.g.) and $0 \% \mathrm{KCl}$ (Expts. 1,2), $0.078 \%$ x.g. and $0.024 \% \mathrm{KCl}$ (Expts. 3,4), $0.078 \%$ x.g. and $0.047 \% \mathrm{KCl}$ (Expts. $5,6,7), 0.156 \%$ x.g. and $0.024 \% \mathrm{KCl}$ (Expts. 8,9).

the best fit of the raw data (without resolution bars) for the changing filament radius to the dimensional form of $H(\tau)$ (3.15). There are three fitting parameters: $a(0), \dot{\varepsilon}(0)$, and $\lambda$, (a fourth parameter $h(0)$ is determined from measurements). For convenience we fit the data to the parameters $\lambda, \delta$ (which is a composition of $a(0)$ and $\lambda$ ) and $\alpha$ (which is a composition of $a(0), \lambda$, and $\dot{\varepsilon}(0))$ under the restriction that $\alpha$ satisfies (3.14). Figure 11 shows the results for nine experiments where the data are denoted by pluses and the fit by the solid line; the corresponding concentrations of xanthan gum and $\mathrm{KCl}$, and the fitted parameter values for $\delta, \lambda$, and $\dot{\varepsilon}(0)$ are reported in Table 1 . We report $\delta$, $\lambda$, and $\dot{\varepsilon}(0)$ since they are most useful for interpreting the results. In all of the experiments, the exact solution models well the thinning of the liquid filament over all time.

The relaxation time determined from fitting to (3.15) is on the order of tens of milliseconds in all of the experiments, with an average value of $26.4 \mathrm{~ms}$. In contrast, the relaxation time of these fluids measured in shear rheology is on the order of tens of seconds. There appears to be no correlation between the fitted relaxation time and the polymer or salt concentrations, as observed in shear rheology measurements. For each experiment, there is a small range of allowable relaxation times on the order of tens of milliseconds accounting for the experimental resolution; though this range is not large enough to account for the difference with the shear rheology measurements. We thus conclude, when measured in extension, the relaxation time of these fluids is nearly constant. 
Table 1. The fitted parameters comparing the exact solution $H(\tau)$ (3.15) and the asymptotic solution (3.26) to experimental data. The fluids are solutions of xanthan gum and $\mathrm{KCl}$ mixed in 80:20 glycerol/water

\begin{tabular}{cccccccc}
\hline \hline Expt. No. & {$[$ X.G. $](\%)$} & {$[\mathrm{KCl}](\%)$} & $\delta$ & $\lambda(\mathrm{s})$ & $\dot{\varepsilon}(0)\left(\mathrm{s}^{-1}\right)$ & $H_{\infty}$ & $\tau_{\infty}$ \\
\hline 1 & 0.078 & 0 & 1.83 & 0.032 & 55.5 & 0.41 & -1.20 \\
2 & 0.078 & 0 & 1.34 & 0.031 & 64.0 & 0.65 & -0.59 \\
3 & 0.078 & 0.024 & 4.64 & 0.043 & 75.1 & 0.06 & -2.84 \\
4 & 0.078 & 0.024 & 1.49 & 0.020 & 127.3 & 0.41 & -0.83 \\
5 & 0.078 & 0.047 & 1.10 & 0.022 & 91.4 & 1.40 & -0.15 \\
6 & 0.078 & 0.047 & 2.54 & 0.028 & 64.8 & 0.68 & -0.39 \\
7 & 0.078 & 0.047 & 1.99 & 0.027 & 69.2 & 0.44 & -0.90 \\
8 & 0.156 & 0.024 & 4.28 & 0.025 & 52.0 & 0.08 & -2.50 \\
9 & 0.156 & 0.024 & 1.67 & 0.010 & 71.1 & 1.32 & -0.74 \\
\hline \hline
\end{tabular}

The exact solution (3.15) for the extensional flow of a viscoelastic fluid provides a single analytic function that connects the effects of the initial stress $T_{z z}$ along the filament to the eventual approach to the Newtonian scaling. The Deborah number $\delta$ represents the balance of this stress through the characteristic stretch rate $\sqrt{a_{0} / \rho}$ relative to $\lambda$. During a transient period, the fluid filament is strongly viscoelastic if either $E_{0}>\delta^{2} / 2$ or $\delta>\delta^{*}$ as explained in $\S 3.5$. This criterion is satisfied for all of the experiments, as shown in Figure 9 , where the parameters from the experiments are denoted by solid dots. This explains why $H(\tau)$ does not scale as $\tau^{-1 / 2}$ initially in the experiments, as shown in Figure 11 . In the weakly viscoelastic case $\left(E_{0}<\delta^{2} / 2\right)$, the filament should behave "Newtonian-like" for all time. We did not study the weakly viscoelastic limit in our experiments, and instead leave this for a future study.

After a transient period, the exact solution for the filament radius transitions to "Newtonian-like" behavior, as explained in $\S 3.3$. We examine more closely this transition and the long-time behavior of the filament motion in Figure 12. The data from Experiment 1 for the nondimensional filament radius $H(\tau)$ vs. nondimensional time are shown in Figure 12(a) (pluses) and the solid line is a fit using (3.15) with $\delta=1.83, \lambda=3.2 \times 10^{-2} \mathrm{~s}$, and $\dot{\varepsilon}(0)=55.5 \mathrm{~s}^{-1}$. The fluid is $0.078 \%$ xanthan gum in $80: 20$ glycerol/water, the same fluid as that shown in Figure 2. The log-log plot of the data shows that after a transient period $\left(\tau \simeq 2\right.$ ), the filament radius decays as $\tau^{-1 / 2}$ (dot-dashed); this scaling is consistent with the asymptotic behavior of $H(\tau)$ (3.26) for $\tau>-\tau_{\infty}$ denoted by the dashed line. Fitting the portion of the data set that is predicted by (3.22) to be in the "Newtonian-like" regime to (3.26), we find $H_{\infty}=0.41$ and $\tau_{\infty}=-1.20$; these values are consistent with the predictions given by (3.22) and (3.27). The values of $H_{\infty}$ and $\tau_{\infty}$ for all of the experiments are reported in Table 1.

From a physical standpoint, the observed transition in $H$ from non-Newtonian behavior to Newtonian scaling can be understood in terms of the response of the polymer molecules to the stretch rate. It is well known that viscoelastic effects delay the breakup of filaments and jets [32, 38], where the stabilization is attributed to the polymer's resistance to extensional deformations comparable to $1 / 2 \lambda[23,6,11]$. In terms of nondimensional 

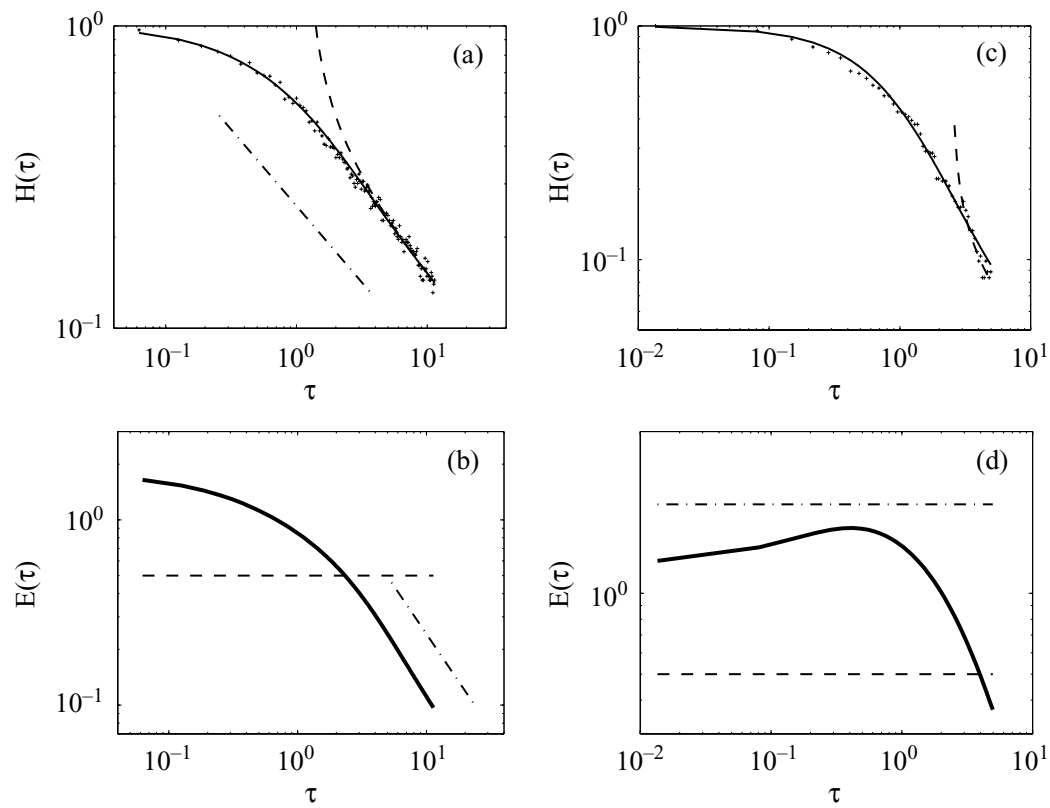

Figure 12. (a) Comparison of the exact solution for $H(\tau)$ (solid) with measurements (pluses) for $0.078 \%$ xanthan gum and $0 \% \mathrm{KCl}$ (Expt. 1) showing the asymptotic scaling $\tau^{-1 / 2}$ (see text); (b) the calculated $E(\tau)$ (solid) determined from the fit for $H(\tau)$ to Expt. 1, showing the asymptotic scaling $\tau^{-1}$ (dot-dashed) and the transition stretch rate $E_{\text {tr }}=\frac{1}{2}$ (dashed); (c) comparison of exact solution for $H(\tau)$ (solid) with measurements (pluses) for $0.156 \%$ xanthan gum and $0.024 \% \mathrm{KCl}$ (Expt. 8), and (d) the calculated $E(\tau)$ determined from the fit for $H(\tau)$ to Expt. 8 (solid), $E_{\text {cr }}=\delta / 2$ (dot-dashed), and the transition stretch rate $E_{\mathrm{tr}}=\frac{1}{2}$ (dashed).

quantities, if the stretch rate of the flow exceeds $1 / 2$, then the polymer molecules stretch from their coiled state, while below this critical value the molecules relax back to a coiled configuration; $E_{\mathrm{tr}} \approx 1 / 2$ corresponds to the stretch-coil transition of the polymer molecules $[23,11,28]$.

Figure 12(b) shows that the calculated value of $E$ (solid line), determined from the fitted parameters of Experiment 1 for $H$, monotonically decreases. This behavior is expected since $E_{0}=1.78>\delta / 2$ (3.30). The dashed line $E_{\mathrm{tr}}=1 / 2$ in Figure 12(b) represents the transition from polymer stretching $\left(E>E_{\mathrm{tr}}\right)$ to relaxing $\left(E<E_{\mathrm{tr}}\right)$. Comparison of Figures 12(a) and (b) show that this transition corresponds roughly to the beginning of the $\tau^{-1 / 2}$ scaling for the filament radius $(\tau \sim 2)$. Thus, at small times the delayed thinning of the filament is an elastic effect caused by the stretched polymer molecules, while at larger times the molecules relax and the fluid behavior is "Newtonian-like".

While monotonic decreasing of the stretch rate was common in the experiments, we did observe one case where the stretch rate increased over a transient period, as shown in Figure 12. The fluid is $0.156 \%$ xanthan gum and $0.024 \% \mathrm{KCl}$ in $80: 20$ glycerol/water (Experiment 8). Figure 12(c) shows measurements of the filament radius (pluses), the nonlinear fit of the data to (3.15) (solid line), and the approach to the asymptotic "Newtonian-like" behavior (3.26) (dashed line). Figure 12(d) shows the corresponding 
stretch rate (solid line) determined from the fitted parameters for $H(\tau)$ to Experiment 8 . Since $E_{0}<\delta / 2$ (dot-dashed), the stretch rate initially increases, as explained in $\S 3.5$. This is a transient effect, so that eventually the stretch rate decreases and dips below the stretch-to-coil transition stretch rate (dashed).

\section{Conclusions}

We have developed an analytic formulation for the free boundary problem of a spatially uniform filament stretching in a gravity-driven purely extensional flow and have provided a condition that must be satisfied for axisymmetric solutions to exist. This approach can be applied to Newtonian or viscoelastic fluids by introducing an appropriate constitutive model, in particular, we obtain the known exact solution for a Newtonian filament [22]. For more complex constitutive models, if one assumes a power series expansion for the stress field, the free boundary problem can be simplified to a coupled system of ODEs. For the Upper Convected Maxwell and Oldroyd-B models we have found an exact solution describing the stretch rate and filament thickness which converges to the Newtonian scaling as $t \rightarrow \infty$. Based on the exact solution, we identify two regimes of dynamical behavior called the weakly- and strongly-viscoelastic limits. For the weakly-viscoelastic case, corresponding to low Deborah numbers, the dynamics are comparable to Newtonian behavior for all times and yield an effective increase in the filament thickness relative to a Newtonian fluid. In the strongly-viscoelastic case, initial transient dynamics are not comparable to Newtonian behavior and the effective filament thickness decreases with increasing Deborah number. Furthermore, in the weakly- (strongly-) viscoelastic limit, as elasticity increases the time-delay before the solution converges to the Newtonian scaling decreases (increases). The viscoelastic solution correctly predicts the time-dependence of the changing filament radius in measurements with semi-dilute (strongly-viscoelastic) polymer solutions. As $t \rightarrow \infty$, observations of the filament thickness follow the Newtonian scaling $1 / \sqrt{t}$. The transition from viscoelastic to Newtonian scaling in the filament thickness is coupled to a stretch-to-coil transition of the polymer molecules. We do not address the stability of the viscoelastic solution to spatial perturbations, instead leaving this for a future investigation. In principle, the analytic approach presented here could be extended to other constitutive models, including those that are nonlinear in stress.

\section{Acknowledgments}

We would like to thank M. G. Forest, N. Handzy, H. Segur and C. Topaz for many helpful discussions. LBS acknowledges the support of the National Science Foundation VIGRE Postdoctoral Fellowship Program (DMS-9983320). AB acknowledges the support of the A. P. Sloan Foundation and the National Science Foundation (CAREER Award DMR-0094167). TW acknowledges the support of the A. P. Sloan Foundation and the National Science Foundation (CAREER Award DMS-0239125).

\section{References}

[1] Abramowitz, M. \& Stegun, I. A. (1964) Handbook of Mathematical Functions. USGPO.

[2] Amarouchene, Y., Bonn, D., Meunier, J. \& Kellay, H. (2001) Inhibition of the finite-time singularity during droplet fission of a polymeric fluid. Phys. Rev. Lett. 86, 3558-3561. 
[3] Anna, S. L. \& McKinley, G. H. (2001) Elasto-capillary thinning and breakup of model elastic liquids. J. Rheol. 45, 115-138.

[4] Bechtel, S. E., Forest, M. G. \& Lin, K. J. (1992) Closure to all orders in 1-D models for slender viscoelastic free jets: An integrated theory for axisymmetric, torsionless flows. Stability Appl. Anal. Continuous Media, 2, 59-100.

[5] Bird, R. B., Armstrong, R. C. \& Hassager, O. (1987) Dynamics of Polymeric Liquids: Vol. 1. Wiley.

[6] Bousfield, D. W., Keunings, R., Marrucci, G. \& Denn, M. M. (1986) Nonlinear analysis of the surface tension driven breakup of viscoelastic filaments. J. Non-Newtonian Fluid Mech. 21, 79-97.

[7] Brenner, M. P., Eggers, J., Joseph, K., Nagel, S. R. \& Shi, X. D. (1997) Breakdown of scaling in droplet fission at high Reynolds number. Phys. Fluids, 9, 1573-1590.

[8] Chang, H.-C., Demekhin, E. A. \& Kalaidin, E. (1999) Iterated stretching of viscoelastic jets. Phys. Fluids, 11, 1717-1737.

[9] Christanti, Y. \& Walker, L. M. (2001) Surface tension driven jet break up of strain-hardening polymer solutions. J. Non-Newtonian Fluid Mech. 100, 9-26.

[10] Clasen, C., Eggers, J., Fontelos, M. A., Li, J. \& McKinley, G. H. The beads-on-string structure of viscoelastic threads. J. Fluid Mech. (submitted).

[11] Denn, M. M., Petrie, C. J. S. \& Avenas, P. (1975) Mechanics of steady spinning of a viscoelastic liquid. AIChE J. 21, 791-799.

[12] Dewynne, J. N. \& Wilmott, P. (1993) Slender axisymmetric fluid jets. Math. Comput. Modelling, 18, 69-82.

[13] EgGers, J. \& Dupont, T. F. (1994) Drop formation in a one-dimensional approximation of the Navier-Stokes equation. J. Fluid Mech. 262, 205-221.

[14] Eggers, J. (1995) Theory of drop formation. Phys. Fluids, 7, 941-953.

[15] EgGers, J. (1997) Nonlinear dynamics and breakup of free-surface flows. Rev. Mod. Phys. 69, 865-929.

[16] Entov, V. M. \& Hinch, E. J. (1997) Effect of a spectrum of relaxation times on the capillary thinning of a filament of elastic liquid. J. Non-Newtonian Fluid Mech. 72, $31-53$.

[17] Frankel, I. \& Weihs, D. (1985) Stability of a capillary jet with linearly increasing axial velocity. J. Fluid Mech. 155, 289-307.

[18] Frankel, I. \& Weihs, D. (1987) Influence of viscosity on the capillary instability of a stretching jet. J. Fluid Mech. 185, 361-383.

[19] Fuller, G. G., Cathey, C. A., Hubbard, B. \& Zebrowski, B. E. (1987) Extensional viscosity measurements for low-viscosity fluids. J. Rheol. 31, 235-249.

[20] Goldin, M., Yerushalmi, J., Pfeffer, R. \& Shinnar, R. (1969) Breakup of a laminar capillary jet of a viscoelastic fluid. J. Fluid Mech. 38, 689-711.

[21] Henderson, D. M., Pritchard, W. G. \& Smolka, L. B. (1997) On the pinch-off of a pendant drop of viscous fluid. Phys. Fluids 9, 3188-3200.

[22] Henderson, D., Segur, H., Smolka, L. B. \& Wadati, M. (2000) The motion of a falling liquid filament. Phys. Fluids 12, 550-565.

[23] Hinch, E. J. (1994) Uncoiling a polymer molecule in a strong extensional flow. J. Non-Newtonian Mech. 54, 209-230.

[24] Ince, E. L. (1944) Ordinary Differential Equations. Dover.

[25] Jones, W. M., Hudson, N. E. \& Ferguson, J. (1990) The extensional properties of M1 obtained from the stretching of a filament by a falling pendant drop. J. Non-Newtonian Fluid Mech. 35, 263-276.

[26] JosePh, D. D. (1990) Fluid Dynamics of Viscoelastic Liquids. Springer-Verlag.

[27] KeILler, R. A. (1992) Extending filaments of an Oldroyd fluid. J. Non-Newtonian Fluid Mech. 42, 37-48.

[28] Keller, A. \& Odell, J. A. (1985) The extensibility of macromolecules in solution; a new focus for macromolecular science. Colloid Polym. Sci. 263, 181-201. 
[29] Khagram, M., Gupta, R. K. \& Sridhar, T. (1985) Extensional flow of xanthan gum solutions. J. Rheol. 29, 191-207.

[30] Kowalewski, T. A. (1996) On the separation of droplets from a liquid jet. Fluid Dyn. Res. 17, $121-145$.

[31] Larson, R. G. (1988) Constitutive Equations for Polymer Melts and Solutions. Butterworths.

[32] Markovich, P. \& Renardy, M. (1985) A finite difference study of the stretching and break-up of filaments of polymer solutions. J. Non-Newtonian Fluid Mech. 17, 13-22.

[33] Mun, R. P., Byars, J. A. \& Boger, D. V. (1998) The effects of polymer concentration and molecular weight on the breakup of laminar capillary jets. J. Non-Newtonian Fluid Mech. 74, 285-297.

[34] Nussinovitch, A. (1997) Hydrocolloid Applications. Blackie Academic \& Professional.

[35] Olagunju, D. O. (1999) A 1-D theory for extensional deformation of a viscoelastic filament under exponential stretching. J. Non-Newtonian Fluid Mech. 87, 27-46.

[36] Papageorgiou, D. T. (1995) On the breakup of viscous liquid threads. Phys. Fluids, 7, 15291544.

[37] Peregrine, D. H., Shoker, G. \& Symon, A. (1990) The bifurcation of liquid bridges. J. Fluid Mech. 212, 25-39.

[38] Renardy, M. (1995) A numerical study of the asymptotic evolution and breakup of Newtonian and viscoelastic jets. J. Non-Newtonian Fluid Mech. 59, 267-282.

[39] Renardy, M. (2000) Mathematical Analysis of Viscoelastic Flows. SIAM.

[40] Rochefort, W. E. \& Middleman, S. (1987) Rheology of Xanthan Gum: Salt, Temperature, and Strain Effects in Oscillatory and Steady Shear Experiments. J. Rheol. 31, 337-369.

[41] Schulkes, R. M. S. M. (1994) The evolution and bifurcation of a pendant drop. J. Fluid Mech. 278, 83-100.

[42] Schultz, W. W. \& Davis, S. H. (1982) One-dimensional liquid fibers. J. Rheol. 26, 331-345.

[43] Shi, X. D., Brenner, M. P. \& Nagel, S. R. (1994) A cascade of structure in a drop falling from a faucet. Science $\mathbf{2 6 5}, 219-222$.

[44] Smolka, L. B. \& Belmonte, A. (2000) Effects of salt on filament dynamics during drop pinch-off in xanthan gum solutions. Phys. Fluids (submitted).

[45] Smolka, L. B. (2002) On the motion of Newtonian and non-Newtonian liquid filaments: stretching, beading, blistering, pinching. PhD thesis, Pennsylvania State University.

[46] Sostarecz, M. C. \& Belmonte, A. (2003) Motion and shape of a viscoelastic drop falling through a viscous fluid. J. Fluid Mech. 497, 235-252.

[47] Sridhar, T., Tirtaatmadja, V., Nguyen, D. A. \& Gupta, R. K. (1991) Measurement of extensional viscosity of polymer solutions. J. Non-Newtonian Fluid Mech. 40, 271-280.

[48] Sridhar, T., Nguyen, D. A. \& Fuller, G. G. (2000) Birefringence and stress growth in uniaxial extension of polymer solutions. J. Non-Newtonian Mech. 90, 299-315.

[49] Trouton, F. T. (1906) On the coefficient of viscous traction and its relation to that of viscosity. Proc. Roy. Soc. Lond. A77, 426-440.

[50] Wang, Q., Forest, M. G. \& Bechtel, S. E. (1995) Modeling and computation of the onset of failure in polymeric liquid filaments. J. Non-Newtonian Fluid Mech. 58, 97-129.

[51] Wilkes, E. D., Phillips, S. D. \& Basaran, O. A. (1999) Computational and experimental analysis of dynamics of drop formation. Phys. Fluids 11, 3577-3598.

[52] Witelski, T. P. \& Bernoff, A. J. (1998) Self-similar asymptotics for linear and nonlinear diffusion equations. Stud. Appl. Math. 100, 153-193.

[53] Zhang, W. W. \& Lister, J. R. (1999) Similarity solutions for capillary pinch-off in fluids of differing viscosity. Phys. Rev. Lett. 83, 1151-1154.

[54] Zhang, X. \& Basaran, O. A. (1995) An experimental study of dynamics of drop formation. Phys. Fluids 7, 1184-1203. 